LTH 1123

\title{
Symmetric point 4-point functions at one loop in QCD
}

\author{
J.A. Gracey, \\ Theoretical Physics Division, \\ Department of Mathematical Sciences, \\ University of Liverpool, \\ P.O. Box 147, \\ Liverpool, \\ L69 3BX, \\ United Kingdom.
}

\begin{abstract}
We evaluate the quartic ghost and quark Green's functions as well as the gluonghost, gluon-quark and ghost-quark 4-point functions of Quantum Chromodynamics at one loop at the fully symmetric point in a linear covariant gauge. Similar expressions for the analogous Green's functions in Quantum Electrodynamics are also provided.
\end{abstract}




\section{Introduction.}

Quantum Chromodynamics (QCD) has served as the central quantum field theory which describes the force between the partons of the nucleons. By partons we mean the quarks which are bound together by the force carrying quantum which is the gluon. The theory has been paramount in describing physics at the high energy scales used in particle accelerators such as the Large Hadron Collider. What is less clear is the connection between the QCD field theory at low energy and the properties of hadrons. The latter are the observed states of nature and if QCD is to explain these particles at a fundamental level then the field theory needs to be solved at low energy. This is not a straightforward task. At high energy QCD is asymptotically free, 1, 2], in that the strength of the coupling constant decreases with the increase in energy. Thus one can apply perturbative techniques to carry out highly precise computations. For example, such multiloop methods have recently been employed to determine the $\beta$-function of QCD to five loops in [3] which was subsequently verified in [4]. As the energy scale decreases the strength of the coupling constant increases and therefore the perturbative approximation ceases to be of use for studying infrared properties of QCD. For instance, one would have to have a large number of terms in a series which would then have to be summed to get anywhere near meaningful results at lower energies. This is of course if one overlooks the fact that purely non-perturbative phenomena could become relevant at lower energies. By non-perturbative we mean contributions which are non-analytic functions of the coupling constant and therefore not accessible from perturbation theory. These issues aside the major gap in our understanding is the actual mechanism by which quarks condense to form hadrons and why quarks and gluons are confined and not observed as free entities in nature. To tackle these problems in a theoretical framework requires techniques which are valid beyond the perturbative regime. The two dominant methods are lattice gauge theory and Schwinger-Dyson equations. The former is a numerically intense approach while the latter extends beyond perturbation theory by systematically solving all the $n$-point Green's functions. As there are an infinite number of such equations at some point an approximation has to be made so that they truncate to a finite set of equations which is manageable.

Much progress has been made in QCD using Schwinger-Dyson equations over the last forty years or so with the focus being on the 2- and 3-point functions. One of the truncations which is ordinarily made in the Schwinger-Dyson approach is to neglect contributions from 4-point vertex functions or 4-point Green's function kernels. This has turned out in general to be a reasonable assumption. However, with the improvement in computational tools to provide more precise insight into the infrared behaviour of QCD it is now the situation where the neglect of 4-point vertex functions needs to be re-examined. Indeed in the Schwinger-Dyson study of [5] the effect of omitting the quartic gluon vertex function has been numerically quantified. More recently there has been interest in investigating the unquenching effects of the gluonic 3-point function through what is termed the swordfish diagram which involves the quark-gluon 4-point function, [6]. The model for that Green's function in the Schwinger-Dyson context grew out of ideas developed in [7]. While such an approach could in some sense be viewed as a first analysis the model suffers from lack of input from explicit field theory calculations. For instance, the solution of any set of Schwinger-Dyson equations has to agree with high energy perturbative results of the same Green's function. For 2- and 3-point functions this has been clearly tested at length and verified over many years. For 4-point functions the situation is less clear. This is mainly because the full off-shell 4-point functions are not known even at one loop in QCD. What is available is the gluon 4-point function at one loop at the fully symmetric subtraction point. The original one loop analysis was carried out in [8]. Although that concentrated on the issue of renormalization schemes a more recent analysis was provided in [9] which recorded the 
full 4-point function at the symmetric point including all possible Lorentz tensor channels. This at least gives a benchmark contact point for comparison with a Schwinger-Dyson analysis of the same function.

What is lacking now is the same information for the quark-gluon 4-point function, for instance, as it is now becoming relevant for the complete 3-point vertex function Schwinger-Dyson construction. Therefore, it is the purpose of this article to compile that Green's function at the fully symmetric point in QCD at one loop similar to the gluon 4-point function of [8, 9]. In addition we will carry out the same computation for the other 4-point functions which are the gluon-ghost and ghost-quark 4-point functions as well as the quartic ghost and quark Green's functions. While these latter Green's functions may not be immediately relevant to the discussion of the approximation made in [6, 7] for the gluonic 3-point function they will be relevant for other 3-point vertex functions as well as Schwinger-Dyson studies of 4-point functions. Indeed there has been progress in the latter respect in recent years with the study of the quartic gluon and quartic quark Green's functions. For instance, a non-exhaustive set of articles on these are [10, 11, 12, 13, 14, 15] and [16, 17] respectively. At this point we note that all our computations will be carried out for the canonical linear covariant gauge. In this gauge the five QCD Green's functions considered here have no tree term in contrast to other gauges. For instance, in the nonlinear Curci-Ferrari gauge, [18, there is a quartic ghost interaction in the Lagrangian. While our main focus is in the linear covariant gauge, as this is the one relevant for Schwinger-Dyson analyses, the tools provided here can in principle be applied to the Curci-Ferrari gauge as well as other gauges. Equally as the 4-point functions can be studied in Quantum Electrodynamics (QED) we will provide the same information for the quartic photon and electron Green's functions as well as the photon-electron 4-point function. This will be a separate evaluation since our QCD computations, like [9], will be performed purely in the $S U\left(N_{c}\right)$ colour group, where $N_{c}$ is the number of colours, for which the abelian limit cannot be taken straightforwardly in a 4-point analysis.

The article is organized as follows. The formalism, notation and technical details of how the computations were carried out are discussed in the next section. The subsequent sections record respectively the results for the five Green's functions of QCD and the three of QED. We provide conclusions in section 5. An appendix records the various Lorentz tensor bases which we used to decompose the various Green's functions into.

\section{Formalism.}

We begin our analysis by discussing the general formalism used to evaluate the Green's functions we are interested in at the fully symmetric point. This will be based for the most part on the approach used in 9] to determine the gluon 4-point function which in the notation we will

use was $\left\langle A_{\mu}^{a}(p) A_{\nu}^{b}(q) A_{\sigma}^{c}(r) A_{\rho}^{d}(-p-q-r)\right\rangle$. Our convention will be that the final field of the Green's function is the one where the conservation of energy-momentum is implemented as $p, q$ and $r$ are the external momentum. The restriction of these momenta to the symmetric point is defined by, [8],

$$
p^{2}=q^{2}=r^{2}=-\mu^{2}, p q=p r=q r=\frac{1}{3} \mu^{2}
$$

which implies that the Mandelstam variables given by

$$
s=\frac{1}{2}(p+q)^{2}, \quad t=\frac{1}{2}(q+r)^{2}, u=\frac{1}{2}(p+r)^{2}
$$


are equivalent since

$$
s=t=u=-\frac{4}{3} \mu^{2}
$$

where $\mu$ is an arbitrary mass scale. As the gluon and quark fields carry Lorentz and spinor indices respectively and these fields as well as the Faddeev-Popov ghost are labelled with colour group indices the decomposition of each Green's function is not straightforward. Therefore we need a systematic way of extracting the structure of the Green's functions which accommodates this complication and allows us to perform the one loop evaluation. Before discussing this we need to record explicitly the setup of the various Green's functions in the form in which they were computed which are

$$
\begin{aligned}
\left.\left\langle\bar{c}^{a}(p) c^{b}(q) A_{\sigma}^{c}(r) A_{\rho}^{d}(-p-q-r)\right\rangle\right|_{\mathrm{symm}} & =\left.\Sigma_{A c \sigma \rho}^{a b c d}(p, q, r)\right|_{\mathrm{symm}} \\
\left.\left\langle\bar{\psi}(p) \psi(q) A_{\sigma}^{c}(r) A_{\rho}^{d}(-p-q-r)\right\rangle\right|_{\mathrm{symm}} & =\left.\Sigma_{A \psi \sigma \rho}^{c d}(p, q, r)\right|_{\mathrm{symm}} \\
\left.\left\langle\bar{c}^{a}(p) c^{b}(q) \bar{c}^{c}(r) c^{d}(-p-q-r)\right\rangle\right|_{\mathrm{symm}} & =\left.\Sigma_{c c}^{a b c d}(p, q, r)\right|_{\mathrm{symm}} \\
\left.\left\langle\bar{c}^{a}(p) c^{b}(q) \bar{\psi}(r) \psi(-p-q-r)\right\rangle\right|_{\mathrm{symm}} & =\left.\Sigma_{c \psi}^{a b}(p, q, r)\right|_{\mathrm{symm}} \\
\left.\left\langle\bar{\psi}_{J}^{j \beta}(p) \psi_{I \alpha}^{i}(q) \bar{\psi}_{K}^{k \gamma}(r) \psi_{L \delta}^{l}(-p-q-r)\right\rangle\right|_{\mathrm{symm}} & =\left.\Sigma_{\psi \psi}(p, q, r)\right|_{\mathrm{symm}}
\end{aligned}
$$

and

$$
\left.\left\langle A_{\mu}^{a}(p) A_{\nu}^{b}(q) A_{\sigma}^{c}(r) A_{\rho}^{d}(-p-q-r)\right\rangle\right|_{\mathrm{symm}}=\left.\Sigma_{A A \mu \nu \sigma \rho}^{a b c d}(p, q, r)\right|_{\mathrm{symm}} .
$$

We include the quartic gluon one for completeness and for reference to the parallel computation in [9] but have appended the label $A A$ to the definition used in [9]. The restriction denoted by symm indicates evaluation with the values of (2.1). In each Green's function we have included various indices. These are the adjoint colour indices $1 \leq a \leq\left(N_{c}^{2}-1\right)$, colour spinor indices $1 \leq I \leq N_{c}$, Lorentz indices $\mu$, spinor indices $1 \leq \alpha \leq 4$, and flavour indices $1 \leq i \leq N_{f}$ where $N_{f}$ is the number of massless quarks and our colour group generators are $T_{I J}^{a}$. The colour and Lorentz indices are included in the shorthand notation for each Green's function and cannot be treated disjointly. To ease discussion we will denote the general form of these by $\left.\Sigma_{\mathcal{L} \mu_{1} \ldots \mu_{n_{l}}}^{a_{1} \ldots a_{n_{c}}}(p, q, r)\right|_{\text {symm }}$ where $\mathcal{L}$ is the label defining the Green's function. The convention for the label $\mathcal{L}$ is that it involves a pair of fields. Each field of the pair represents two of the fields in the actual 4-point function in a compact and obvious fashion. We have not included the various spinor and flavour indices in the shorthand for $\left.\Sigma_{\psi \psi}(p, q, r)\right|_{\text {symm }}$ as aside from being cumbersome the computation of this Green's function was carried out in a different way to the other five and will be treated differently in the discussion. In defining the ranges of the colour group indices we have assumed at the outset that we are working with the colour group $S U\left(N_{c}\right)$. As discussed in [8, 9] this eases the computation of the underlying Feynman graphs and in particular the treatment of box graphs. We will use the colour group algorithm given in [9] for the evaluation of $\left.\Sigma_{A c \sigma \rho}^{a b c d}(p, q, r)\right|_{\text {symm }}$ and refer the interested reader to that for more details. However, it will turn out that the final form of this Green's function will involve various rank 4 colour tensors which are $f_{4}^{a b c d}, f_{4}^{a d b c}$ and $d_{A}^{a b c d}$ where

$$
f_{4}^{a b c d}=f^{a b e} f^{c d e} \quad, \quad d_{A}^{a b c d}=\frac{1}{6} \operatorname{Tr}\left(T_{A}^{a} T_{A}^{(b} T_{A}^{c} T_{A}^{d)}\right)
$$

and $f^{a b c}$ are the $S U\left(N_{c}\right)$ structure constants. The tensor $d_{A}^{a b c d}$ is totally symmetric in its indices and was introduced in [19]. The subscript $A$ on the group generators indicates that they are in the adjoint representation. These are the three combinations of tensors which arose in the gluon 4-point function for the graphs involving fields in the adjoint representation, 9]. We exclude 
$f_{4}^{a c d b}$ as it is not independent due to the Jacobi identity. In [9] other rank 4 tensors arose in the quartic gluon 4-point function due to closed loops of quarks. As there is no direct coupling of the quark to the ghost these tensors do not occur in the quartic ghost or gluon-ghost 4-point functions at one loop. For the remaining Green's function we use the purely $S U\left(N_{c}\right)$ relation for the group generators

$$
T_{I J}^{a} T_{K L}^{a}=\frac{1}{2}\left[\delta_{I L} \delta_{K J}-\frac{1}{N_{c}} \delta_{I J} \delta_{K L}\right]
$$

which was useful for graphs involving quarks in the remaining Green's functions. Ordinarily the evaluation of 2- and 3-point functions in perturbative QCD is carried out for a general colour group without resorting to relations such as (2.7) which are group specific. This means that in those cases expressions for QED can be deduced by taking the abelian limit where, for instance, the structure constants are formally set to zero. As we will be using (2.7) for the 4-point functions of (2.4) the QED limit of the resulting expressions cannot be found by this process. Instead to obtain these a direct evaluation has to be carried out. Given the formalism we will use this is not a difficult task and we will provide the one loop structure of the Green's functions

$$
\begin{aligned}
\left.\left\langle A_{\mu}(p) A_{\nu}(q) A_{\sigma}(r) A_{\rho}(-p-q-r)\right\rangle\right|_{\mathrm{symm}} & =\left.\Sigma_{A A \mu \nu \sigma \rho}^{\mathrm{QED}}(p, q, r)\right|_{\mathrm{symm}} \\
\left.\left\langle\bar{\psi}(p) \psi(q) A_{\sigma}(r) A_{\rho}(-p-q-r)\right\rangle\right|_{\mathrm{symm}} & =\left.\Sigma_{A \psi \sigma \rho}^{\mathrm{QED}}(p, q, r)\right|_{\mathrm{symm}} \\
\left.\left\langle\bar{\psi}^{j \beta}(p) \psi_{\alpha}^{i}(q) \bar{\psi}^{k \gamma}(r) \psi_{\delta}^{l}(-p-q-r)\right\rangle\right|_{\mathrm{symm}} & =\left.\Sigma_{\psi \psi}^{\mathrm{QED}}(p, q, r)\right|_{\mathrm{symm}}
\end{aligned}
$$

where fields without colour group labels are either the photon or electron of QED. A superscript label is included to avoid confusion of the results with those of QCD. Included in this set of Green's functions is the photon 4-point function and there are no ghost interactions in a linear covariant gauge fixed QED Lagrangian which we use here.

For the moment we will concentrate our discussion on the evaluation of each of the QCD Green's functions. Where necessary we will append comments on differences with the QED evaluation. In the case of QCD the number of different independent colour group tensors which can appear is rather small and is determined from the rules noted above. However, what is more involved is the treatment of the Lorentz structure. In [9] a projection method was used to decompose the gluon 4-point function into the 138 different possible Lorentz structures which could be built from the independent external momenta $p, q$ and $r$ as well as the metric $\eta^{\mu \nu}$. One of the reasons a projection method was necessary rested in the way the individual Feynman graphs of a Green's function were evaluated. Use was made of the Laporta algorithm [20]. This is an integration by parts routine where linear relations are derived between scalar Feynman integrals in $d$-dimensions. These can be solved algebraically in such a way that all the integrals are related to a small set of what is termed master integrals. These are determined by direct methods and hence the whole evaluation of the Green's function can be coded in a fully automatic fashion, [20]. What is key to the application of the Laporta algorithm is writing the Green's function of interest in terms of scalar Feynman integrals. When the Green's function has Lorentz or spinor indices this means they first have to be converted to a scalar form. While we do this with a projection method for most of the Green's functions of interest here, that for the quartic quark one is treated differently. This can best be understood by first considering the construction of the basis for three of the other four Green's functions. By this we mean gluon-ghost, gluon-quark and ghost-quark as the quartic ghost 4-point function does not require a Lorentz decomposition as the ghost is a Lorentz singlet. Of these three two have spinor indices but not that for the gluon-ghost 4-point function. For the Lorentz basis of the latter the rank 2 Lorentz tensors 
of the basis have to be built from the same basic objects as that for the quartic gluonic one. Rather than the 138 possibilities for that rank 4 4-point function the basis for the gluon-ghost 4-point function has 10 independent possibilities. One of these is $\eta^{\mu \nu}$ while the remaining nine correspond to the $3^{2}$ independent products of the three independent external momenta with different Lorentz indices. While this is a simple analysis for a quarkless 4-point function the basis of objects for a tensor basis has to be increased when there are quark external legs. This is because one has to allow for spinor indices which are carried by the unit spinor matrix and the $\gamma$-matrices. In the case of the latter the $\gamma$-matrices can have free Lorentz indices or be contracted with external momenta. Additionally there is the complication of amending the decomposition to allow for the use of dimensional regularization which we use throughout. In dimensionally regularizing in $d=4-2 \epsilon$ dimensions, where $\epsilon$ is the regularizing parameter, the $\gamma$-algebra ceases to be finite dimensional and instead is infinite dimensional. To accommodate this both within the actual computation and the construction of the tensor basis we use the generalized $\gamma$-matrices denoted by $\Gamma_{(n)}^{\mu_{1} \ldots \mu_{n}},[21,22,23,24,25]$, and defined as the antisymmetric product of the $\gamma$-matrices satisfying the $d$-dimensional Clifford algebra

$$
\left\{\gamma^{\mu}, \gamma^{\nu}\right\}=2 \eta^{\mu \nu}
$$

as

$$
\Gamma_{(n)}^{\mu_{1} \ldots \mu_{n}}=\gamma^{\left[\mu_{1}\right.} \ldots \gamma^{\left.\mu_{n}\right]}
$$

A factor of $1 / n$ ! is understood in the antisymmetrization. We will regard $\gamma^{\mu}$ and $\Gamma_{(1)}^{\mu}$ as being synonymous throughout. A major benefit of this choice for the $d$-dimensional $\gamma$-matrices is that the $\Gamma_{(n)}$-matrices naturally partition the infinite dimensional spinor space since the generalized trace is

$$
\operatorname{tr}\left(\Gamma_{(m)}^{\mu_{1} \ldots \mu_{m}} \Gamma_{(n)}^{\nu_{1} \ldots \nu_{n}}\right) \propto \delta_{m n} I^{\mu_{1} \ldots \mu_{m} \nu_{1} \ldots \nu_{n}} .
$$

The quantity $I^{\mu_{1} \ldots \mu_{m} \nu_{1} \ldots \nu_{n}}$ represents the unit matrix in the $\Gamma_{(n)}$-space where there is no sum on $m$ or $n$. As an aside on the use of dimensional regularization we use the scale $\mu$ of (2.1) to ensure that the coupling constant in the regularized theory is dimensionless. This is the same convention as was used in [9].

More importantly this partitioning gives a natural way of constructing a Lorentz basis for the gluon-quark and ghost-quark Green's functions. First one determines which partitions of the $\Gamma_{(n)}$-basis will be present. In the case of these two Green's functions $n$ will be odd as we are using massless quarks and the spinor line will be the product of $\gamma$-matrices from the quarkgluon vertex Feynman rule or the quark propagator. As there are no Lorentz indices on the ghost-quark 4-point function then the Lorentz indices of the contributing $\Gamma_{(n)}$-matrices have to be contracted with the independent external momenta. By this reasoning there are only four possibilities with $n$ being no larger than 3 . Three derive from the contraction of $\Gamma_{(1)}^{\mu}$ with each external momenta and the final one involves $\Gamma_{(3)}^{\mu \nu \sigma}$ contracted with the three different momenta due to the antisymmetric property. For the gluon-quark 4-point function the presence of two Lorentz indices increases the number of possible tensors in comparison. Also this means that for the $\Gamma_{(n)}$-matrix partition $n$ can be at most five since $\Gamma_{(5)}^{\mu \nu p q r}$ can be present. For the lower partitions we constructed the basis by considering the possible cases for $n=1$ and 3 separately. When a Lorentz index on the $\Gamma_{(n)}$-matrix is contracted with an external momentum the free Lorentz indices can be carried by the external momenta themselves and the metric tensor. For the $n=1$ partition there are 36 tensors but only 31 for $n=3$ and 1 for $n=5$. The full set is recorded in the Appendix using the notation we will elaborate on shortly. Equipped with this knowledge of how the tensors for the Lorentz basis are constructed it is easy to see that its application to the quartic quark Green's function is not straightforward. For that 4-point function one has two independent spinor strings and the spinor sector of the Lorentz basis 
will formally be $\Gamma_{(m)} \otimes \Gamma_{(n)}$ where $m$ and $n$ are not necessarily equal and the tensor product reflects the two independent spinor threads. The imbalance is possible due to contractions with independent external momenta as will become evident. To construct the basis for this Green's function in generality is not practical. For instance, at high enough loop order one would have to allow in principle for possible tensors in the basis such as $\Gamma_{(7)}^{\mu \nu \sigma \rho p q r} \otimes \Gamma_{(5)}^{\mu \nu \sigma \rho q}$ for example and similar higher partitions. This is virtually impossible to accommodate in the projection method. Therefore for the quartic quark 4-point function we have followed the variation presented in [26]. In [26] and [27] a similar problem arose but with three spinor strings. To effect the Laporta algorithm scalar integrals were produced by stripping all the $\gamma$-algebra from the individual Feynman integrals and then decomposing the underlying Lorentz integrals into scalar integrals coupled with Lorentz tensors involving only the metric and external momenta. We refer the interested reader to [26] for an example of the more technical aspects of this point which is straightforward to implement at one loop. Beyond that order it becomes less efficient than the projection method. However, after the Lorentz tensor integrals have been decomposed the $\gamma$ matrices are recontracted and then these $\gamma$-matrix strings are decomposed into the $\Gamma_{(n)}$-matrix basis which is achieved via the recursive relations, [23, 24, 25],

$$
\begin{aligned}
\Gamma_{(n)}^{\mu_{1} \ldots \mu_{n}} \gamma^{\nu} & =\Gamma_{(n+1)}^{\mu_{1} \ldots \mu_{n} \nu}+\sum_{r=1}^{n}(-1)^{n-r} \eta^{\mu_{r} \nu} \Gamma_{(n-1)}^{\mu_{1} \ldots \mu_{r-1} \mu_{r+1} \ldots \mu_{n}} \\
\gamma^{\nu} \Gamma_{(n)}^{\mu_{1} \ldots \mu_{n}} & =\Gamma_{(n+1)}^{\nu \mu_{1} \ldots \mu_{n}}+\sum_{r=1}^{n}(-1)^{r-1} \eta^{\mu_{r} \nu} \Gamma_{(n-1)}^{\mu_{1} \ldots \mu_{r-1} \mu_{r+1} \ldots \mu_{n}} .
\end{aligned}
$$

The outcome of this detour for the quartic quark Green's function is to arrive at the point where that 4-point function is written in terms of scalar Feynman integrals.

For the other three Lorentz 4-point functions the actual projection matrices which act on each Green's function to produce the analogous scalar Feynman integrals needs to be constructed. We can treat each of the gluon-ghost, gluon-quark and ghost-quark 4-point functions simultaneously if we write the decomposition as

$$
\left.\Sigma_{\mathcal{L}}^{a_{1} \ldots a_{n_{c}} \mu_{1} \ldots \mu_{n_{l}}}(p, q, r)\right|_{\text {symm }}=\left.\sum_{k=1}^{n_{p}} \mathcal{P}_{\mathcal{L}(k)}^{\mu_{1} \ldots \mu_{n_{l}}}(p, q, r) \Sigma_{\mathcal{L}(k)}^{a_{1} \ldots a_{n_{c}}}(p, q, r)\right|_{\mathrm{symm}}
$$

where $\mathcal{P}_{\mathcal{L}(k)}^{\mu_{1} \ldots \mu_{n_{l}}}(p, q, r)$ are the Lorentz tensors of the respective bases and $\Sigma_{\mathcal{L}(k)}^{a_{1} \ldots a_{n_{c}}}(p, q, r)$ are the scalar amplitudes associated with the label $k$ denoting an element of the tensor basis. Each set is given in the Appendix. The indexing set of the projectors has dimension $n_{p}$ and the number of Lorentz indices is $n_{l}$. We retain the colour group indices on these amplitudes for ease at the moment but the explicit colour dependent object will be factored off in the final expression of the Green's function. It is the Lorentz tensors which are provided in the Appendix and which we use subsequently. To construct the projection matrix we first find the matrix $\mathcal{N}_{\mathcal{L} k k^{\prime}}$ defined by

$$
\mathcal{N}_{\mathcal{L} k k^{\prime}}=\left.\mathcal{P}_{\mathcal{L}(k) \mu_{1} \ldots \mu_{n_{l}}}(p, q, r) \mathcal{P}_{\mathcal{L}\left(k^{\prime}\right)}^{\mu_{1} \ldots \mu_{n_{l}}}(p, q, r)\right|_{\text {symm }}
$$

which is symmetric in the Lorentz tensor basis labels $k$ and $k^{\prime}$. Its elements are polynomials in $d$ and its inverse is $\mathcal{M}_{\mathcal{L} k k^{\prime}}$ which is the projector on the Green's function. In other words the amplitudes in the Lorentz decomposition are given by

$$
\left.\Sigma_{A A(k)}^{a b c d}(p, q, r)\right|_{\mathrm{symm}}=\left.\mathcal{M}_{A A k k^{\prime}}\left(\mathcal{P}_{A A\left(k^{\prime}\right)}^{\mu \nu \sigma \rho}(p, q, r)\left\langle A_{\mu}^{a}(p) A_{\nu}^{b}(q) A_{\sigma}^{c}(r) A_{\rho}^{d}(-p-q-r)\right\rangle\right)\right|_{\mathrm{symm}}
$$

for the quartic gluon Green's function where there is a sum over the label $k^{\prime}$. A summary of the data for the parameters is given in Table 1 where $n_{s}$ is the number of independent spinor 
threads for each Green's function. We have included the same data for the quartic gluon case for completeness as well as for reference to [9]. The parallel data for the QED analysis is also included for comparison. No information appears in the $n_{c}$ column in that case.

\begin{tabular}{|c||c|c|c|c|c|}
\hline Green's function & $n_{p}$ & $n_{c}$ & $n_{l}$ & $n_{\gamma}$ & Graphs \\
\hline$A_{\mu}^{a} A_{\nu}^{b} A_{\sigma}^{c} A_{\rho}^{d}$ & 138 & 4 & 4 & 0 & 24 \\
$\bar{c}^{a} c^{b} A_{\sigma}^{c} A_{\rho}^{d}$ & 10 & 4 & 2 & 0 & 7 \\
$\bar{\psi} \psi A_{\sigma}^{c} A_{\rho}^{d}$ & 68 & 2 & 2 & 1 & 7 \\
$\bar{c}^{a} c^{b} \bar{c}^{c} c^{d}$ & 1 & 4 & 0 & 0 & 4 \\
$\bar{c}^{a} c^{b} \bar{\psi} \psi$ & 4 & 2 & 0 & 1 & 2 \\
$\bar{\psi}_{J}^{j \beta} \psi_{I \alpha}^{i} \bar{\psi}_{K}^{k \gamma} \psi_{L \delta}^{l}$ & $(168)$ & 0 & 0 & 2 & 4 \\
\hline$A_{\mu} A_{\nu} A_{\sigma} A_{\rho}$ & 138 & - & 4 & 0 & 6 \\
$\bar{\psi} \psi A_{\sigma} A_{\rho}$ & 68 & - & 2 & 1 & 2 \\
$\bar{\psi}^{j \beta} \psi_{\alpha}^{i} \bar{\psi}^{k \gamma} \psi_{\delta}^{l}$ & $(72)$ & - & 0 & 2 & 4 \\
\hline
\end{tabular}

Table 1. Summary data for each of the Green's functions in QCD (top) and QED (bottom).

The final column in Table 1 indicates the number of one loop Feynman graphs contributing to each Green's function. The absence of a ghost in a QED linear covariant gauge fixing reduces the number of Feynman graphs needed to be computed directly in that case. This leads us to briefly discuss the technical machinery used to evaluate all the 4-point functions to one loop. The Feynman graphs are generated automatically by the QGRAF package, [28], in an electronic format which can be adapted to the symbolic manipulation language Form, [29, 30], which we have used to write the above algorithm in. We have encoded both the projection method and the method to write the quark 4-point function as scalar Feynman integrals in separate FORM modules after all the Lorentz, spinor and colour group indices have been mapped into the QGRAF generated graphs. In each of the approaches scalar products of the internal momentum and the external momenta are rewritten in terms of the propagators of the integral. As this is a one loop computation for a 4-point function there are no irreducible scalar products although there are three different box integral families at one loop. By integral family we mean the core topologies, with the constraints (2.1) implemented, in the syntax of the Laporta algorithm, [20]. To effect the Laporta algorithm we used its implementation in the early version of the package REDUZE, [31, which was also used in [9]. The package has the feature that the relations giving the reduction to the master integrals can be converted to Form notation. So we have written these in a Form module and included it within our automatic Feynman graph computation. Once all the projections and integration by parts for a graph have been applied the final stage is the substitution of the various master integrals. For the symmetric point, (2.1), their explicit values were recorded in [9]. However, these were special cases of the more general evaluation of one loop massless triangle and box integrals derived in [32, 33, 34, 35] for all external legs fully off-shell.

This completes the technical aspects of the tools we used to implement the algorithm to evaluate the Green's functions. One minor feature of our computations which differs from the quartic gluon Green's function is that that 4-point function needed to be renormalized. This is not the case here for the QED and the QCD 4-point functions of (2.8) and (2.4) as they have no corresponding interaction in the original QED or QCD Lagrangians with a linear covariant gauge fixing. So they all have to be finite without the introduction of any new renormalization constant. This provides a minor check on our computation and we indeed found that all the one loop Green's functions were finite. At higher loop the wave function renormalization for the external legs of the Green's functions and the conversion of the bare parameters to renormalized 
variables would have to be included. At one loop this is not necessary. It is also worth noting that these comments have to be modified if one uses a different gauge fixing to the canonical linear gauge fixing. For instance, in the nonlinear Curci-Ferrari gauge, [18], there is a quartic ghost interaction in the gauge fixed Lagrangian. Equally in the maximal abelian gauge, [36, 37, 38], there are gluon-ghost 4-point interactions in addition to quartic ghost ones. Of course in both these gauges the final form of the various Green's functions we consider here would be structurally different.

\section{QCD results.}

We are now in a position to discuss the results of the computations. As there are no Lorentz structures in the ghost 4-point function we can illustrate main aspects of the form of the results for this and the other Green's function. For an arbitrary linear covariant gauge we find

$$
\begin{aligned}
\left.\Sigma_{c c}^{a b c d}(p, q, r)\right|_{\text {symm }}= & {\left[\frac{31}{320}-\frac{51}{160} \alpha+\frac{71}{320} \alpha^{2}+\frac{177}{1600} \ln \left(\frac{4}{3}\right)-\frac{107}{800} \ln \left(\frac{4}{3}\right) \alpha\right.} \\
& +\frac{37}{1600} \ln \left(\frac{4}{3}\right) \alpha^{2}-\frac{1003}{51200} \Phi_{1}\left(\frac{9}{16}, \frac{9}{16}\right)+\frac{423}{25600} \Phi_{1}\left(\frac{9}{16}, \frac{9}{16}\right) \alpha \\
& +\frac{3357}{51200} \Phi_{1}\left(\frac{9}{16}, \frac{9}{16}\right) \alpha^{2}-\frac{1}{32} \Phi_{1}\left(\frac{3}{4}, \frac{3}{4}\right)+\frac{5}{64} \Phi_{1}\left(\frac{3}{4}, \frac{3}{4}\right) \alpha \\
& \left.-\frac{11}{64} \Phi_{1}\left(\frac{3}{4}, \frac{3}{4}\right) \alpha^{2}\right] f_{4}^{a c b d} N_{c} a+O\left(a^{2}\right)
\end{aligned}
$$

where $a=g^{2} /\left(16 \pi^{2}\right)$ is the renormalized coupling constant and $\alpha$ is the renormalized gauge fixing parameter of the linear covariant gauge fixing we use throughout. The Landau gauge corresponds to $\alpha=0$. The function $\Phi_{1}(x, y)$ corresponds with $\Phi(x, y)$ of [35] and involves the dilogarithm function $\operatorname{Li}_{2}(z)$. The definition is

$$
\Phi_{1}(x, y)=\frac{1}{\lambda}\left[2 \operatorname{Li}_{2}(-\rho x)+2 \operatorname{Li}_{2}(-\rho y)+\ln \left(\frac{y}{x}\right) \ln \left(\frac{(1+\rho y)}{(1+\rho x)}\right)+\ln (\rho x) \ln (\rho y)+\frac{\pi^{2}}{3}\right]
$$

where

$$
\lambda(x, y)=\sqrt{\Delta_{G}} \quad, \quad \rho(x, y)=\frac{2}{[1-x-y+\lambda(x, y)]}
$$

and

$$
\Delta_{G}(x, y)=x^{2}-2 x y+y^{2}-2 x-2 y+1
$$

which is the Gram determinant. The function $\Phi_{1}(x, y)$ appears throughout all the Green's functions and arises in two contexts in each computation. They occur in masters which are in effect a triangle or 3-point function and when there is a pure box graph. The triangle master emerges either as a consequence of the original Feynman graph being a triangle before integration by parts reduction or within the reduction of an original box graph. Each function is reflected differently in the analytic form of the Green's function at the symmetric point as $\Phi_{1}\left(\frac{3}{4}, \frac{3}{4}\right)$ and $\Phi_{1}\left(\frac{9}{16}, \frac{9}{16}\right)$. The reason for the different arguments is due to the symmetric point values of the squared external momenta, 32, 33, 34, 35. More specifically the two functions are related to the Clausen function $\mathrm{Cl}(\theta)$ by, [32, 33,

$$
\begin{aligned}
\Phi_{1}\left(\frac{3}{4}, \frac{3}{4}\right) & =\sqrt{2}\left[2 \mathrm{Cl}_{2}\left(2 \cos ^{-1}\left(\frac{1}{\sqrt{3}}\right)\right)+\mathrm{Cl}_{2}\left(2 \cos ^{-1}\left(\frac{1}{3}\right)\right)\right] \\
\Phi_{1}\left(\frac{9}{16}, \frac{9}{16}\right) & =\frac{4}{\sqrt{5}}\left[2 \mathrm{Cl}_{2}\left(2 \cos ^{-1}\left(\frac{2}{3}\right)\right)+\mathrm{Cl}_{2}\left(2 \cos ^{-1}\left(\frac{1}{9}\right)\right)\right] .
\end{aligned}
$$


While we have given the quartic ghost function explicitly we give a flavour of the remaining functions by recording their numerical values. We note that the full analytic form of each of the 4-point functions given here are provided in an attached data file. For the numerical versions we note that

$$
\Phi_{1}\left(\frac{3}{4}, \frac{3}{4}\right)=2.832045 \quad, \quad \Phi_{1}\left(\frac{9}{16}, \frac{9}{16}\right)=3.403614 .
$$

With these values it is straightforward to provide the Green's functions in a compact form. All the cases we record explicitly are for the $S U(3)$ colour group. First, for comparison the quartic ghost function is

$$
\left.\Sigma_{c c}^{a b c d}(p, q, r)\right|_{\mathrm{symm}}=-\left[0.079434+0.239204 \alpha+0.105202 \alpha^{2}\right] f_{4}^{a c b d} a+O\left(a^{2}\right)
$$

in an arbitrary gauge. As the remaining 4-point functions are much larger the remaining Green's functions are all given in the Landau gauge. For the gluon-ghost 4-point function we have

$$
\begin{aligned}
\left.\Sigma_{A c \sigma \rho}^{a b c d}(p, q, r)\right|_{\text {symm }}=[ & 0.069633 \mathcal{P}_{A c(1)}-0.018972 \mathcal{P}_{A c(2)}-0.566599 \mathcal{P}_{A c(3)} \\
+ & 0.091060 \mathcal{P}_{A c(4)}-0.445930 \mathcal{P}_{A c(5)}+0.101697 \mathcal{P}_{A c(6)} \\
+ & 0.211729 \mathcal{P}_{A c(7)}-0.048584 \mathcal{P}_{A c(8)}-0.169253 \mathcal{P}_{A c(9)} \\
+ & \left.0.042475 \mathcal{P}_{A c(10)}\right] d_{A}^{a b c d} a \\
+ & {\left[0.192709 \mathcal{P}_{A c(1)}+1.000682 \mathcal{P}_{A c(2)}-0.506828 \mathcal{P}_{A c(3)}\right.} \\
& +1.115084 \mathcal{P}_{A c(4)}-0.632469 \mathcal{P}_{A c(5)}-0.811897 \mathcal{P}_{A c(6)} \\
& -0.697495 \mathcal{P}_{A c(7)}+0.595902 \mathcal{P}_{A c(8)}-0.853676 \mathcal{P}_{A c(9)} \\
& \left.+0.024346 \mathcal{P}_{A c(10)}\right] f_{4}^{a b c d} a \\
+ & 0.385419 \mathcal{P}_{A c(1)}+0.314724 \mathcal{P}_{A c(2)}-1.013359 \mathcal{P}_{A c(3)} \\
& +0.543528 \mathcal{P}_{A c(4)}-1.376360 \mathcal{P}_{A c(5)}-0.048277 \mathcal{P}_{A c(6)} \\
& +0.180527 \mathcal{P}_{A c(7)}-0.494836 \mathcal{P}_{A c(8)}-0.131835 \mathcal{P}_{A c(9)} \\
& \left.+0.048692 \mathcal{P}_{A c(10)}\right] f_{4}^{a d b c} a+O\left(a^{2}\right) .
\end{aligned}
$$

This form is indicative of the other Green's function in that the colour group structure is more involved. We have not implemented the Jacobi identity for instance to rearrange the expression as it was not clear if this would lead to any simplifications. Moreover, with two different external fields with one external momentum expressed in terms of the others the symmetry structure is not apparent. For the gluon-quark 4-point function not every Lorentz structure is present in each colour group channel as

$$
\begin{aligned}
\left.\Sigma_{A \psi \sigma \rho}^{c d}(p, q, r)\right|_{\text {symm }}=[ & 0.051996 \mathcal{P}_{A \psi(1)}-0.051996 \mathcal{P}_{A \psi(2)}+0.051996 \mathcal{P}_{A \psi(4)} \\
& -0.051996 \mathcal{P}_{A \psi(5)}+0.665903 \mathcal{P}_{A \psi(7)}-0.665903 \mathcal{P}_{A \psi(8)} \\
& +0.052679 \mathcal{P}_{A \psi(10)}+0.625933 \mathcal{P}_{A \psi(11)}+0.728409 \mathcal{P}_{A \psi(12)} \\
& -0.516160 \mathcal{P}_{A \psi(13)}+0.054818 \mathcal{P}_{A \psi(14)}+1.821741 \mathcal{P}_{A \psi(15)} \\
& -0.262442 \mathcal{P}_{A \psi(16)}+0.241748 \mathcal{P}_{A \psi(17)}+1.264728 \mathcal{P}_{A \psi(18)} \\
& -0.054818 \mathcal{P}_{A \psi(19)}+0.516160 \mathcal{P}_{A \psi(20)}-1.821741 \mathcal{P}_{A \psi(21)} \\
& -0.625933 \mathcal{P}_{A \psi(22)}-0.052679 \mathcal{P}_{A \psi(23)}-0.728409 \mathcal{P}_{A \psi(24)} \\
& -0.241748 \mathcal{P}_{A \psi(25)}+0.262442 \mathcal{P}_{A \psi(26)}-1.264728 \mathcal{P}_{A \psi(27)} \\
& -0.798761 \mathcal{P}_{A \psi(28)}+1.436663 \mathcal{P}_{A \psi(29)}-0.798761 \mathcal{P}_{A \psi(30)}
\end{aligned}
$$




$$
\begin{aligned}
& -1.436663 \mathcal{P}_{A \psi(31)}+0.798761 \mathcal{P}_{A \psi(32)}+0.798761 \mathcal{P}_{A \psi(33)} \\
& -0.798761 \mathcal{P}_{A \psi(34)}+0.798761 \mathcal{P}_{A \psi(35)}-1.302123 \mathcal{P}_{A \psi(37)} \\
& -1.302123 \mathcal{P}_{A \psi(38)}-2.604247 \mathcal{P}_{A \psi(39)}-0.376857 \mathcal{P}_{A \psi(40)} \\
& +0.376857 \mathcal{P}_{A \psi(41)}-0.764445 \mathcal{P}_{A \psi(43)}-1.272049 \mathcal{P}_{A \psi(44)} \\
& -1.016975 \mathcal{P}_{A \psi(45)}+1.272049 \mathcal{P}_{A \psi(46)}+0.764445 \mathcal{P}_{A \psi(47)} \\
& +1.016975 \mathcal{P}_{A \psi(48)}+0.130747 \mathcal{P}_{A \psi(49)}-0.130747 \mathcal{P}_{A \psi(50)} \\
& -0.252531 \mathcal{P}_{A \psi(52)}+0.255073 \mathcal{P}_{A \psi(53)}-1.016975 \mathcal{P}_{A \psi(54)} \\
& -0.255073 \mathcal{P}_{A \psi(55)}+0.252531 \mathcal{P}_{A \psi(56)}+1.016975 \mathcal{P}_{A \psi(57)} \\
& -0.258102 \mathcal{P}_{A \psi(59)}-0.343551 \mathcal{P}_{A \psi(60)}-0.258102 \mathcal{P}_{A \psi(61)} \\
& +0.343551 \mathcal{P}_{A \psi(62)}+0.258102 \mathcal{P}_{A \psi(63)}+0.258102 \mathcal{P}_{A \psi(64)} \\
& \left.-0.258102 \mathcal{P}_{A \psi(65)}+0.258102 \mathcal{P}_{A \psi(66)}-1.701880 \mathcal{P}_{A \psi(68)}\right] \delta^{c d} a \\
& +\left[0.029665 \mathcal{P}_{A \psi(1)}+2.316733 \mathcal{P}_{A \psi(2)}+2.344135 \mathcal{P}_{A \psi(3)}\right. \\
& +0.027402 \mathcal{P}_{A \psi(4)}+2.314469 \mathcal{P}_{A \psi(5)}+2.344135 \mathcal{P}_{A \psi(6)} \\
& -0.066898 \mathcal{P}_{A \psi(7)}-3.976814 \mathcal{P}_{A \psi(8)}-4.043712 \mathcal{P}_{A \psi(9)} \\
& +0.838848 \mathcal{P}_{A \psi(10)}+2.284266 \mathcal{P}_{A \psi(11)}+2.172543 \mathcal{P}_{A \psi(12)} \\
& +0.727540 \mathcal{P}_{A \psi(13)}+1.579463 \mathcal{P}_{A \psi(14)}+4.425123 \mathcal{P}_{A \psi(15)} \\
& +0.450090 \mathcal{P}_{A \psi(16)}+1.223824 \mathcal{P}_{A \psi(17)}+2.342749 \mathcal{P}_{A \psi(18)} \\
& -0.912599 \mathcal{P}_{A \psi(19)}+0.410488 \mathcal{P}_{A \psi(20)}-3.979770 \mathcal{P}_{A \psi(21)} \\
& -1.005854 \mathcal{P}_{A \psi(22)}+0.533701 \mathcal{P}_{A \psi(23)}-1.021505 \mathcal{P}_{A \psi(24)} \\
& -0.754487 \mathcal{P}_{A \psi(25)}+0.724932 \mathcal{P}_{A \psi(26)}-4.308456 \mathcal{P}_{A \psi(27)} \\
& -2.639334 \mathcal{P}_{A \psi(28)}+1.641136 \mathcal{P}_{A \psi(29)}-2.860845 \mathcal{P}_{A \psi(30)} \\
& -2.810777 \mathcal{P}_{A \psi(31)}+1.092665 \mathcal{P}_{A \psi(32)}+0.871154 \mathcal{P}_{A \psi(33)} \\
& -2.836861 \mathcal{P}_{A \psi(34)}+0.895138 \mathcal{P}_{A \psi(35)}-1.965707 \mathcal{P}_{A \psi(36)} \\
& -4.789443 \mathcal{P}_{A \psi(37)}-2.415426 \mathcal{P}_{A \psi(38)}-7.204870 \mathcal{P}_{A \psi(39)} \\
& +0.630272 \mathcal{P}_{A \psi(40)}+1.068829 \mathcal{P}_{A \psi(41)}-2.643495 \mathcal{P}_{A \psi(42)} \\
& +1.033312 \mathcal{P}_{A \psi(43)}-0.384262 \mathcal{P}_{A \psi(44)}-0.562489 \mathcal{P}_{A \psi(45)} \\
& +0.717403 \mathcal{P}_{A \psi(46)}-0.210354 \mathcal{P}_{A \psi(47)}+1.420985 \mathcal{P}_{A \psi(48)} \\
& -2.259213 \mathcal{P}_{A \psi(49)}-4.165986 \mathcal{P}_{A \psi(50)}-1.784999 \mathcal{P}_{A \psi(51)} \\
& -1.631339 \mathcal{P}_{A \psi(52)}-0.703582 \mathcal{P}_{A \psi(53)}-1.420985 \mathcal{P}_{A \psi(54)} \\
& +0.178227 \mathcal{P}_{A \psi(55)}+1.595801 \mathcal{P}_{A \psi(56)}+0.562489 \mathcal{P}_{A \psi(57)} \\
& +2.334275 \mathcal{P}_{A \psi(58)}-0.490947 \mathcal{P}_{A \psi(59)}-0.439694 \mathcal{P}_{A \psi(60)} \\
& -0.493727 \mathcal{P}_{A \psi(61)}-0.273666 \mathcal{P}_{A \psi(62)}-0.269298 \mathcal{P}_{A \psi(63)} \\
& -0.272078 \mathcal{P}_{A \psi(64)}+0.021890 \mathcal{P}_{A \psi(65)}+0.243539 \mathcal{P}_{A \psi(66)} \\
& \left.-0.250188 \mathcal{P}_{A \psi(67)}+0.319089 \mathcal{P}_{A \psi(68)}\right] T^{c} T^{d} a \\
& +\left[-2.316733 \mathcal{P}_{A \psi(1)}-0.029665 \mathcal{P}_{A \psi(2)}-2.344135 \mathcal{P}_{A \psi(3)}\right. \\
& -2.314469 \mathcal{P}_{A \psi(4)}-0.027402 \mathcal{P}_{A \psi(5)}-2.344135 \mathcal{P}_{A \psi(6)} \\
& +3.976814 \mathcal{P}_{A \psi(7)}+0.066898 \mathcal{P}_{A \psi(8)}+4.043712 \mathcal{P}_{A \psi(9)} \\
& -0.533701 \mathcal{P}_{A \psi(10)}+1.005854 \mathcal{P}_{A \psi(11)}+1.021504 \mathcal{P}_{A \psi(12)} \\
& -0.410488 \mathcal{P}_{A \psi(13)}+0.912599 \mathcal{P}_{A \psi(14)}+3.979770 \mathcal{P}_{A \psi(15)} \\
& -0.724932 \mathcal{P}_{A \psi(16)}+0.754487 \mathcal{P}_{A \psi(17)}+4.308456 \mathcal{P}_{A \psi(18)}
\end{aligned}
$$




$$
\begin{aligned}
& -1.579463 \mathcal{P}_{A \psi(19)}-0.727540 \mathcal{P}_{A \psi(20)}-4.425123 \mathcal{P}_{A \psi(21)} \\
& -2.284266 \mathcal{P}_{A \psi(22)}-0.838848 \mathcal{P}_{A \psi(23)}-2.172543 \mathcal{P}_{A \psi(24)} \\
& -1.223824 \mathcal{P}_{A \psi(25)}-0.450090 \mathcal{P}_{A \psi(26)}-2.342749 \mathcal{P}_{A \psi(27)} \\
& -1.092665 \mathcal{P}_{A \psi(28)}+2.810777 \mathcal{P}_{A \psi(29)}-0.871154 \mathcal{P}_{A \psi(30)} \\
& -1.641136 \mathcal{P}_{A \psi(31)}+2.639334 \mathcal{P}_{A \psi(32)}+2.860845 \mathcal{P}_{A \psi(33)} \\
& -{ }{ } .895138 \mathcal{P}_{A \psi(34)}+2.836861 \mathcal{P}_{A \psi(35)}+1.965707 \mathcal{P}_{A \psi(36)} \\
& -2.415426 \mathcal{P}_{A \psi(37)}-4.789443 \mathcal{P}_{A \psi(38)}-7.204870 \mathcal{P}_{A \psi(39)} \\
& -1.068829 \mathcal{P}_{A \psi(40)}-0.630272 \mathcal{P}_{A \psi(41)}+2.643495 \mathcal{P}_{A \psi(42)} \\
& +0.210354 \mathcal{P}_{A \psi(43)}-0.717403 \mathcal{P}_{A \psi(44)}-1.420985 \mathcal{P}_{A \psi(45)} \\
& +0.384262 \mathcal{P}_{A \psi(46)}-1.033312 \mathcal{P}_{A \psi(47)}+0.562489 \mathcal{P}_{A \psi(48)} \\
& +4.165986 \mathcal{P}_{A \psi(49)}+2.259213 \mathcal{P}_{A \psi(50)}+1.784999 \mathcal{P}_{A \psi(51)} \\
& -1.595801 \mathcal{P}_{A \psi(52)}-0.178227 \mathcal{P}_{A \psi(53)}-0.562489 \mathcal{P}_{A \psi(54)} \\
& +0.703582 \mathcal{P}_{A \psi(55)}+1.631339 \mathcal{P}_{A \psi(56)}+1.420985 \mathcal{P}_{A \psi(57)} \\
& -2.334275 \mathcal{P}_{A \psi(58)}+0.269298 \mathcal{P}_{A \psi(59)}+0.273666 \mathcal{P}_{A \psi(60)} \\
& +0.272078 \mathcal{P}_{A \psi(61)}+0.439694 \mathcal{P}_{A \psi(62)}+0.490947 \mathcal{P}_{A \psi(63)} \\
& +0.493727 \mathcal{P}_{A \psi(64)}-0.243539 \mathcal{P}_{A \psi(65)}-0.021890 \mathcal{P}_{A \psi(66)} \\
& \left.+0.250188 \mathcal{P}_{A \psi(67)}+0.319089 \mathcal{P}_{A \psi(68)}\right] T^{d} T^{c} a+O\left(a^{2}\right)
\end{aligned}
$$

where the group generators are present. A degree of symmetry can be observed. For instance, in the first colour channel quite a few of the Lorentz channels can be paired asymmetrically. The last projected 4-point function is the ghost-quark 4-point function which is

$$
\begin{aligned}
\left.\Sigma_{c \psi}^{a b}(p, q, r)\right|_{\text {symm }}=[ & \left.0.112015 \mathcal{P}_{c \psi(1)}+0.112015 \mathcal{P}_{c \psi(2)}+0.224031 \mathcal{P}_{c \psi(3)}\right] \delta^{a b} a \\
+ & {\left[0.060233 \mathcal{P}_{c \psi(1)}+0.611859 \mathcal{P}_{c \psi(2)}+0.672092 \mathcal{P}_{c \psi(3)}\right.} \\
& \left.+0.557425 \mathcal{P}_{c \psi(4)}\right] T^{a} T^{b} a \\
+ & {\left[0.611859 \mathcal{P}_{c \psi(1)}+0.060233 \mathcal{P}_{c \psi(2)}+0.672092 \mathcal{P}_{c \psi(3)}\right.} \\
& \left.\quad-0.557425 \mathcal{P}_{c \psi(4)}\right] T^{b} T^{a} a+O\left(a^{2}\right)
\end{aligned}
$$

where the unit matrix in the flavour indices is omitted similar to the previous Green's function. Also the expression is like (3.9) as it carries a remnant of the underlying symmetry of working at the fully symmetric point.

As noted the final Green's function was computed in a different way from the previous four in that there was no projection on to the Lorentz basis due to the presence of two $\gamma$-matrix strings. Instead the one loop computation was performed directly and we found

$$
\begin{aligned}
\left.\Sigma_{\psi \psi}(p, q, r)\right|_{\text {symm }}=[ & -0.082685 \mathcal{P}_{\psi \psi(1)}-0.027899 \mathcal{P}_{\psi \psi(2)}+0.208213 \mathcal{P}_{\psi \psi(3)} \\
& +0.285516 \mathcal{P}_{\psi \psi(4)}+0.126257 \mathcal{P}_{\psi \psi(5)}+0.255432 \mathcal{P}_{\psi \psi(6)} \\
& +0.003043 \mathcal{P}_{\psi \psi(7)}-0.088114 \mathcal{P}_{\psi \psi(8)}-0.114487 \mathcal{P}_{\psi \psi(9)} \\
& +0.038162 \mathcal{P}_{\psi \psi(10)}-0.427903 \mathcal{P}_{\psi \psi(11)}-0.252736 \mathcal{P}_{\psi \psi(12)} \\
& +0.313416 \mathcal{P}_{\psi \psi(13)}+0.290898 \mathcal{P}_{\psi \psi(14)}-0.049365 \mathcal{P}_{\psi \psi(15)} \\
& -0.036201 \mathcal{P}_{\psi \psi(16)}+0.140945 \mathcal{P}_{\psi \psi(17)}+0.164419 \mathcal{P}_{\psi \psi(18)} \\
& -0.255432 \mathcal{P}_{\psi \psi(19)}-0.126257 \mathcal{P}_{\psi \psi(20)}-0.126863 \mathcal{P}_{\psi \psi(21)} \\
& +0.042288 \mathcal{P}_{\psi \psi(22)}-0.208942 \mathcal{P}_{\psi \psi(23)}-0.283331 \mathcal{P}_{\psi \psi(24)}
\end{aligned}
$$


$-0.290898 \mathcal{P}_{\psi \psi(25)}-0.313416 \mathcal{P}_{\psi \psi(26)}+0.003043 \mathcal{P}_{\psi \psi(27)}$

$-0.088114 \mathcal{P}_{\psi \psi(28)}+0.043794 \mathcal{P}_{\psi \psi(29)}+0.144571 \mathcal{P}_{\psi \psi(30)}$

$-0.164419 \mathcal{P}_{\psi \psi(31)}-0.140945 \mathcal{P}_{\psi \psi(32)}-0.039244 \mathcal{P}_{\psi \psi(33)}$

$+0.038749 \mathcal{P}_{\psi \psi(34)}-0.144571 \mathcal{P}_{\psi \psi(35)}-0.043794 \mathcal{P}_{\psi \psi(36)}$

$+0.283331 \mathcal{P}_{\psi \psi(37)}+0.208942 \mathcal{P}_{\psi \psi(38)}+0.038749 \mathcal{P}_{\psi \psi(39)}$

$-0.039244 \mathcal{P}_{\psi \psi(40)}-0.285516 \mathcal{P}_{\psi \psi(41)}-0.208213 \mathcal{P}_{\psi \psi(42)}$

$-0.038749 \mathcal{P}_{\psi \psi(43)}+0.039244 \mathcal{P}_{\psi \psi(44)}+0.252736 \mathcal{P}_{\psi \psi(45)}$

$+0.427903 \mathcal{P}_{\psi \psi(46)}+0.039244 \mathcal{P}_{\psi \psi(47)}-0.038749 \mathcal{P}_{\psi \psi(48)}$

$-0.038162 \mathcal{P}_{\psi \psi(49)}+0.114487 \mathcal{P}_{\psi \psi(50)}-0.036201 \mathcal{P}_{\psi \psi(51)}$

$-0.049365 \mathcal{P}_{\psi \psi(52)}+0.027899 \mathcal{P}_{\psi \psi(53)}+0.082685 \mathcal{P}_{\psi \psi(54)}$

$-0.088114 \mathcal{P}_{\psi \psi(55)}+0.003043 \mathcal{P}_{\psi \psi(56)}-0.088114 \mathcal{P}_{\psi \psi(57)}$

$+0.003043 \mathcal{P}_{\psi \psi(58)}+0.042288 \mathcal{P}_{\psi \psi(59)}-0.126863 \mathcal{P}_{\psi \psi(60)}$

$-0.202468 \mathcal{P}_{\psi \psi(61)}+0.607403 \mathcal{P}_{\psi \psi(62)}-0.054181 \mathcal{P}_{\psi \psi(63)}$

$-0.073883 \mathcal{P}_{\psi \psi(64)}+0.123173 \mathcal{P}_{\psi \psi(65)}-0.369519 \mathcal{P}_{\psi \psi(66)}$

$+0.123173 \mathcal{P}_{\psi \psi(67)}-0.369519 \mathcal{P}_{\psi \psi(68)}-0.607403 \mathcal{P}_{\psi \psi(69)}$

$+0.202468 \mathcal{P}_{\psi \psi(70)}-0.369519 \mathcal{P}_{\psi \psi(71)}+0.123173 \mathcal{P}_{\psi \psi(72)}$

$-0.369519 \mathcal{P}_{\psi \psi(73)}+0.123173 \mathcal{P}_{\psi \psi(74)}-0.073883 \mathcal{P}_{\psi \psi(75)}$

$-0.054181 \mathcal{P}_{\psi \psi(76)}-0.073883 \mathcal{P}_{\psi \psi(77)}-0.054181 \mathcal{P}_{\psi \psi(78)}$

$-0.073883 \mathcal{P}_{\psi \psi(79)}-0.054181 \mathcal{P}_{\psi \psi(80)}-0.369519 \mathcal{P}_{\psi \psi(81)}$

$+0.123173 \mathcal{P}_{\psi \psi(82)}+0.123173 \mathcal{P}_{\psi \psi(83)}-0.369519 \mathcal{P}_{\psi \psi(84)}$

$-0.054181 \mathcal{P}_{\psi \psi(85)}-0.073883 \mathcal{P}_{\psi \psi(86)}-0.054181 \mathcal{P}_{\psi \psi(87)}$

$-0.073883 \mathcal{P}_{\psi \psi(88)}-0.023225 \mathcal{P}_{\psi \psi(89)}-0.031671 \mathcal{P}_{\psi \psi(90)}$

$+0.031671 \mathcal{P}_{\psi \psi(91)}+0.023225 \mathcal{P}_{\psi \psi(92)}-0.043943 \mathcal{P}_{\psi \psi(93)}$

$-0.059922 \mathcal{P}_{\psi \psi(94)}+0.064498 \mathcal{P}_{\psi \psi(95)}-0.241431 \mathcal{P}_{\psi \psi(96)}$

$+0.086469 \mathcal{P}_{\psi \psi(97)}-0.211470 \mathcal{P}_{\psi \psi(98)}+0.059922 \mathcal{P}_{\psi \psi(99)}$

$+0.043943 \mathcal{P}_{\psi \psi(100)}+0.029961 \mathcal{P}_{\psi \psi(101)}+0.021972 \mathcal{P}_{\psi \psi(102)}$

$+0.011984 \mathcal{P}_{\psi \psi(103)}-0.019974 \mathcal{P}_{\psi \psi(104)}+0.029961 \mathcal{P}_{\psi \psi(105)}$

$+0.021972 \mathcal{P}_{\psi \psi(106)}+0.211470 \mathcal{P}_{\psi \psi(107)}-0.086469 \mathcal{P}_{\psi \psi(108)}$

$-0.001997 \mathcal{P}_{\psi \psi(109)}-0.041946 \mathcal{P}_{\psi \psi(110)}+0.019974 \mathcal{P}_{\psi \psi(111)}$

$-0.011984 \mathcal{P}_{\psi \psi(112)}-0.043943 \mathcal{P}_{\psi \psi(113)}-0.059922 \mathcal{P}_{\psi \psi(114)}$

$-0.021972 \mathcal{P}_{\psi \psi(115)}-0.029961 \mathcal{P}_{\psi \psi(116)}+0.059922 \mathcal{P}_{\psi \psi(117)}$

$+0.043943 \mathcal{P}_{\psi \psi(118)}+0.041946 \mathcal{P}_{\psi \psi(119)}+0.001997 \mathcal{P}_{\psi \psi(120)}$

$+0.241431 \mathcal{P}_{\psi \psi(121)}-0.064498 \mathcal{P}_{\psi \psi(122)}-0.021972 \mathcal{P}_{\psi \psi(123)}$

$-0.029961 \mathcal{P}_{\psi \psi(124)}-0.087489 \mathcal{P}_{\psi \psi(125)}+0.052717 \mathcal{P}_{\psi \psi(126)}$

$-0.041477 \mathcal{P}_{\psi \psi(127)}+0.073977 \mathcal{P}_{\psi \psi(128)}-0.105841 \mathcal{P}_{\psi \psi(129)}$

$+0.158228 \mathcal{P}_{\psi \psi(130)}+0.099560 \mathcal{P}_{\psi \psi(131)}+0.073010 \mathcal{P}_{\psi \psi(132)}$

$+0.078300 \mathcal{P}_{\psi \psi(133)}+0.026998 \mathcal{P}_{\psi \psi(134)}+0.021259 \mathcal{P}_{\psi \psi(135)}$

$+0.046012 \mathcal{P}_{\psi \psi(136)}+0.257788 \mathcal{P}_{\psi \psi(137)}-0.032831 \mathcal{P}_{\psi \psi(138)}$

$-0.158228 \mathcal{P}_{\psi \psi(139)}+0.105841 \mathcal{P}_{\psi \psi(140)}+0.018352 \mathcal{P}_{\psi \psi(141)}$

$-0.105511 \mathcal{P}_{\psi \psi(142)}-0.046012 \mathcal{P}_{\psi \psi(143)}-0.021259 \mathcal{P}_{\psi \psi(144)}$

$-0.043943 \mathcal{P}_{\psi \psi(145)}-0.059922 \mathcal{P}_{\psi \psi(146)}+0.059922 \mathcal{P}_{\psi \psi(147)}$ 


$$
\begin{aligned}
& +0.043943 \mathcal{P}_{\psi \psi(148)}-0.008646 \mathcal{P}_{\psi \psi(149)}-0.183811 \mathcal{P}_{\psi \psi(150)} \\
& +0.032831 \mathcal{P}_{\psi \psi(151)}-0.257788 \mathcal{P}_{\psi \psi(152)}+0.183811 \mathcal{P}_{\psi \psi(153)} \\
& +0.008646 \mathcal{P}_{\psi \psi(154)}+0.105511 \mathcal{P}_{\psi \psi(155)}-0.018352 \mathcal{P}_{\psi \psi(156)} \\
& -0.073977 \mathcal{P}_{\psi \psi(157)}+0.041477 \mathcal{P}_{\psi \psi(158)}-0.026998 \mathcal{P}_{\psi \psi(159)} \\
& -0.078300 \mathcal{P}_{\psi \psi(160)}-0.073010 \mathcal{P}_{\psi \psi(161)}-0.099560 \mathcal{P}_{\psi \psi(162)} \\
& -0.052717 \mathcal{P}_{\psi \psi(163)}+0.087489 \mathcal{P}_{\psi \psi(164)}-0.080757 \mathcal{P}_{\psi \psi(165)} \\
& \left.-0.110123 \mathcal{P}_{\psi \psi(166)}+0.110123 \mathcal{P}_{\psi \psi(167)}+0.080757 \mathcal{P}_{\psi \psi(168)}\right] a \\
& +O\left(a^{2}\right)
\end{aligned}
$$

where the colour group dependence is present in each tensor basis element. Again there are remnants of the symmetric point present in the expression. However as this was a direct evaluation it is not clear if all colour channels have a contribution for instance. Beyond one loop, by contrast, we would expect additional Lorentz structures with respect to our use of the $\Gamma_{(n)}$-matrices similar to [26, 27].

\section{QED results.}

Having concentrated on QCD we now turn to the case of QED and record the analogous results for the quartic photon, photon-electron and quartic electron Green's functions. The resulting expressions are not obtained by taking a group theory limit but instead are a direct evaluation. Again for space reasons we present the results numerically in the Landau gauge. First, for the photon 4-point function we have

$$
\begin{aligned}
\left.\Sigma_{A A \mu \nu \sigma \rho}^{\mathrm{QED}}(p, q, r)\right|_{\mathrm{symm}}=[ & -0.716260 \mathcal{P}_{A A(1)}-0.716260 \mathcal{P}_{A A(2)}-0.716260 \mathcal{P}_{A A(3)} \\
& -2.382957 \mathcal{P}_{A A(4)}-4.268597 \mathcal{P}_{A A(5)}+4.502776 \mathcal{P}_{A A(6)} \\
& -4.268597 \mathcal{P}_{A A(7)}-2.382957 \mathcal{P}_{A A(8)}+4.502776 \mathcal{P}_{A A(9)} \\
& -2.217185 \mathcal{P}_{A A(10)}-2.217185 \mathcal{P}_{A A(11)}+2.285591 \mathcal{P}_{A A(12)} \\
& -2.382957 \mathcal{P}_{A A(13)}+4.502776 \mathcal{P}_{A A(14)}-4.268597 \mathcal{P}_{A A(15)} \\
& -2.217185 \mathcal{P}_{A A(16)}+2.285591 \mathcal{P}_{A A(17)}-2.217185 \mathcal{P}_{A A(18)} \\
& -4.268597 \mathcal{P}_{A A(19)}+4.502776 \mathcal{P}_{A A(20)}-2.382957 \mathcal{P}_{A A(21)} \\
& +3.771280 \mathcal{P}_{A A(22)}+1.885640 \mathcal{P}_{A A(23)}+1.885640 \mathcal{P}_{A A(24)} \\
& +1.885640 \mathcal{P}_{A A(25)}-2.382957 \mathcal{P}_{A A(26)}-6.885733 \mathcal{P}_{A A(27)} \\
& +1.885640 \mathcal{P}_{A A(28)}-0.165772 \mathcal{P}_{A A(29)}-2.382957 \mathcal{P}_{A A(30)} \\
& +2.285591 \mathcal{P}_{A A(31)}-2.217185 \mathcal{P}_{A A(32)}-2.217185 \mathcal{P}_{A A(33)} \\
& +4.502776 \mathcal{P}_{A A(34)}-2.382957 \mathcal{P}_{A A(35)}-4.268597 \mathcal{P}_{A A(36)} \\
& +4.502776 \mathcal{P}_{A A(37)}-4.268597 \mathcal{P}_{A A(38)}-2.382957 \mathcal{P}_{A A(39)} \\
& -2.382957 \mathcal{P}_{A A(40)}+1.885640 \mathcal{P}_{A A(41)}-0.165772 \mathcal{P}_{A A(42)} \\
& +1.885640 \mathcal{P}_{A A(43)}+3.771280 \mathcal{P}_{A A(44)}+1.885640 \mathcal{P}_{A A(45)} \\
& -6.885733 \mathcal{P}_{A A(46)}+1.885640 \mathcal{P}_{A A(47)}-2.382957 \mathcal{P}_{A A(48)} \\
& -2.382957 \mathcal{P}_{A A(49)}-0.165772 \mathcal{P}_{A A(50)}+1.885640 \mathcal{P}_{A A(51)} \\
& -6.885733 \mathcal{P}_{A A(52)}-2.382957 \mathcal{P}_{A A(53)}+1.885640 \mathcal{P}_{A A(54)} \\
+ & 1.885640 \mathcal{P}_{A A(55)}+1.885640 \mathcal{P}_{A A(56)}+3.771280 \mathcal{P}_{A A(57)} \\
& -1.307807 \mathcal{P}_{A A(58)}-1.307807 \mathcal{P}_{A A(59)}-1.307807 \mathcal{P}_{A A(60)} \\
& -120
\end{aligned}
$$




$$
\begin{aligned}
& -0.838049 \mathcal{P}_{A A(61)}-0.653903 \mathcal{P}_{A A(62)}-0.653903 \mathcal{P}_{A A(63)} \\
& -0.469758 \mathcal{P}_{A A(64)}-0.838049 \mathcal{P}_{A A(65)}-0.653903 \mathcal{P}_{A A(66)} \\
& -0.653903 \mathcal{P}_{A A(67)}-0.469758 \mathcal{P}_{A A(68)}-0.838049 \mathcal{P}_{A A(69)} \\
& -0.653903 \mathcal{P}_{A A(70)}-0.469758 \mathcal{P}_{A A(71)}-0.653903 \mathcal{P}_{A A(72)} \\
& -0.838049 \mathcal{P}_{A A(73)}-0.469758 \mathcal{P}_{A A(74)}-0.653903 \mathcal{P}_{A A(75)} \\
& -0.653903 \mathcal{P}_{A A(76)}-0.838049 \mathcal{P}_{A A(77)}-0.469758 \mathcal{P}_{A A(78)} \\
& -0.653903 \mathcal{P}_{A A(79)}-0.653903 \mathcal{P}_{A A(80)}-0.838049 \mathcal{P}_{A A(81)} \\
& -0.653903 \mathcal{P}_{A A(82)}-0.469758 \mathcal{P}_{A A(83)}-0.653903 \mathcal{P}_{A A(84)} \\
& -0.653903 \mathcal{P}_{A A(85)}-0.838049 \mathcal{P}_{A A(86)}-0.469758 \mathcal{P}_{A A(87)} \\
& -0.838049 \mathcal{P}_{A A(88)}-0.469758 \mathcal{P}_{A A(89)}-0.653903 \mathcal{P}_{A A(90)} \\
& -0.838049 \mathcal{P}_{A A(91)}-0.653903 \mathcal{P}_{A A(92)}-0.469758 \mathcal{P}_{A A(93)} \\
& -0.838049 \mathcal{P}_{A A(94)}-0.653903 \mathcal{P}_{A A(95)}-0.469758 \mathcal{P}_{A A(96)} \\
& -0.838049 \mathcal{P}_{A A(97)}-0.469758 \mathcal{P}_{A A(98)}-0.653903 \mathcal{P}_{A A(99)} \\
& -0.653903 \mathcal{P}_{A A(100)}-0.838049 \mathcal{P}_{A A(101)}-0.469758 \mathcal{P}_{A A(102)} \\
& +5.472775 \mathcal{P}_{A A(103)}-0.497317 \mathcal{P}_{A A(104)}-2.746924 \mathcal{P}_{A A(105)} \\
& -0.497317 \mathcal{P}_{A A(106)}-0.313172 \mathcal{P}_{A A(107)}+5.656920 \mathcal{P}_{A A(108)} \\
& -0.497317 \mathcal{P}_{A A(109)}+5.472775 \mathcal{P}_{A A(110)}-2.746924 \mathcal{P}_{A A(111)} \\
& -8.717016 \mathcal{P}_{A A(112)}+5.656920 \mathcal{P}_{A A(113)}-0.313172 \mathcal{P}_{A A(114)} \\
& -0.497317 \mathcal{P}_{A A(115)}-2.746924 \mathcal{P}_{A A(116)}+5.472775 \mathcal{P}_{A A(117)} \\
& +5.656920 \mathcal{P}_{A A(118)}-8.717016 \mathcal{P}_{A A(119)}+5.656920 \mathcal{P}_{A A(120)} \\
& +5.472775 \mathcal{P}_{A A(121)}-2.746924 \mathcal{P}_{A A(122)}-0.497317 \mathcal{P}_{A A(123)} \\
& -0.313172 \mathcal{P}_{A A(124)}-0.497317 \mathcal{P}_{A A(125)}-0.313172 \mathcal{P}_{A A(126)} \\
& -2.746924 \mathcal{P}_{A A(127)}+5.472775 \mathcal{P}_{A A(128)}-0.497317 \mathcal{P}_{A A(129)} \\
& +5.656920 \mathcal{P}_{A A(130)}-0.313172 \mathcal{P}_{A A(131)}-0.497317 \mathcal{P}_{A A(132)} \\
& -2.746924 \mathcal{P}_{A A(133)}-0.497317 \mathcal{P}_{A A(134)}+5.472775 \mathcal{P}_{A A(135)} \\
& \left.-0.313172 \mathcal{P}_{A A(136)}+5.656920 \mathcal{P}_{A A(137)}-8.717016 \mathcal{P}_{A A(138)}\right] N_{f} a \\
& +O\left(a^{2}\right)
\end{aligned}
$$

which involves all the possible Lorentz tensor structures which appear in the quartic gluon Green's function of [9]. Unlike the quartic gluon Green's function (4.1) begins at $O(a)$ where in QED $a$ is related to the fine structure constant as opposed to the strong coupling constant. The one loop expression is proportional to the number of massless electrons as the only graphs which contribute at this order are closed electron boxes. The situation for the photon-electron 4-point function has parallels to (4.1) as

$$
\begin{aligned}
\left.\Sigma_{A \psi \sigma \rho}^{\mathrm{QED}}(p, q, r)\right|_{\mathrm{symm}}=[ & 0.368797 \mathcal{P}_{A \psi(1)}-0.368797 \mathcal{P}_{A \psi(2)}+0.368797 \mathcal{P}_{A \psi(4)} \\
& -0.368797 \mathcal{P}_{A \psi(5)}-0.406427 \mathcal{P}_{A \psi(7)}+0.406427 \mathcal{P}_{A \psi(8)} \\
& +0.259319 \mathcal{P}_{A \psi(10)}+0.590103 \mathcal{P}_{A \psi(11)}+0.165609 \mathcal{P}_{A \psi(12)} \\
& -0.978151 \mathcal{P}_{A \psi(13)}-1.099679 \mathcal{P}_{A \psi(14)}-0.247840 \mathcal{P}_{A \psi(15)} \\
& -0.248659 \mathcal{P}_{A \psi(16)}-0.286135 \mathcal{P}_{A \psi(17)}-0.308512 \mathcal{P}_{A \psi(18)} \\
& +1.099679 \mathcal{P}_{A \psi(19)}+0.978151 \mathcal{P}_{A \psi(20)}+0.247840 \mathcal{P}_{A \psi(21)} \\
& -0.590103 \mathcal{P}_{A \psi(22)}-0.259319 \mathcal{P}_{A \psi(23)}-0.165609 \mathcal{P}_{A \psi(24)} \\
& +0.286135 \mathcal{P}_{A \psi(25)}+0.248659 \mathcal{P}_{A \psi(26)}+0.308512 \mathcal{P}_{A \psi(27)}
\end{aligned}
$$




$$
\begin{aligned}
& +0.225463 \mathcal{P}_{A \psi(28)}+1.380267 \mathcal{P}_{A \psi(29)}+0.225463 \mathcal{P}_{A \psi(30)} \\
& -1.380267 \mathcal{P}_{A \psi(31)}-0.225463 \mathcal{P}_{A \psi(32)}-0.225463 \mathcal{P}_{A \psi(33)} \\
& +0.225463 \mathcal{P}_{A \psi(34)}-0.225463 \mathcal{P}_{A \psi(35)}-0.645223 \mathcal{P}_{A \psi(37)} \\
& -0.645223 \mathcal{P}_{A \psi(38)}-1.290446 \mathcal{P}_{A \psi(39)}-0.595466 \mathcal{P}_{A \psi(40)} \\
& +0.595466 \mathcal{P}_{A \psi(41)}-2.486948 \mathcal{P}_{A \psi(43)}-2.603035 \mathcal{P}_{A \psi(44)} \\
& -0.977477 \mathcal{P}_{A \psi(45)}+2.603035 \mathcal{P}_{A \psi(46)}+2.486948 \mathcal{P}_{A \psi(47)} \\
& +0.977477 \mathcal{P}_{A \psi(48)}-0.479380 \mathcal{P}_{A \psi(49)}+0.479380 \mathcal{P}_{A \psi(50)} \\
& +1.509471 \mathcal{P}_{A \psi(52)}+1.625558 \mathcal{P}_{A \psi(53)}-0.977477 \mathcal{P}_{A \psi(54)} \\
& -1.625558 \mathcal{P}_{A \psi(55)}-1.509471 \mathcal{P}_{A \psi(56)}+0.977477 \mathcal{P}_{A \psi(57)} \\
& -0.461758 \mathcal{P}_{A \psi(59)}-0.862883 \mathcal{P}_{A \psi(60)}-0.461758 \mathcal{P}_{A \psi(61)} \\
& +0.862883 \mathcal{P}_{A \psi(62)}+0.461758 \mathcal{P}_{A \psi(63)}+0.461758 \mathcal{P}_{A \psi(64)} \\
& \left.-0.461758 \mathcal{P}_{A \psi(65)}+0.461758 \mathcal{P}_{A \psi(66)}-3.829066 \mathcal{P}_{A \psi(68)}\right] a \\
& +O\left(a^{2}\right) \text {. }
\end{aligned}
$$

However, unlike (4.1) not all the Lorentz tensor structures which are in the gluon-quark basis are used in this Green's function. What is more evident in these two Green's functions is that there are more common coefficients of various tensors unlike the non-abelian case.

Finally, the situation of the quartic electron Green's function is somewhat different from its non-abelian counterpart. This is because of the way we had to perform the computation due to the two independent spinor lines. As a consequence of this way we had to organize the calculation we have not used the same Lorentz tensor basis as (3.11). Instead we have constructed a set of tensors specific to this electron Green's function and the explicit forms are given in the Appendix. The full expression is

$$
\begin{aligned}
\left.\Sigma_{\psi \psi}^{\mathrm{QED}}(p, q, r)\right|_{\mathrm{symm}}=[ & -0.413930 \mathcal{P}_{\psi \psi(1)}^{\mathrm{QED}}+1.365234 \mathcal{P}_{\psi \psi(2)}^{\mathrm{QED}}+0.951304 \mathcal{P}_{\psi \psi(3)}^{\mathrm{QED}} \\
& -0.118477 \mathcal{P}_{\psi \psi(4)}^{\mathrm{QED}}-1.779164 \mathcal{P}_{\psi \psi(5)}^{\mathrm{QED}}+1.779164 \mathcal{P}_{\psi \psi(6)}^{\mathrm{QED}} \\
& -0.236954 \mathcal{P}_{\psi \psi(7)}^{\mathrm{QED}}+0.951304 \mathcal{P}_{\psi \psi(8)}^{\mathrm{QED}}-0.951304 \mathcal{P}_{\psi \psi(9)}^{\mathrm{QED}} \\
& -1.365234 \mathcal{P}_{\psi \psi(10)}^{\mathrm{QED}}-1.779164 \mathcal{P}_{\psi \psi(11)}^{\mathrm{QED}}-0.118477 \mathcal{P}_{\psi \psi(12)}^{\mathrm{QED}} \\
& +0.413930 \mathcal{P}_{\psi \psi(13)}^{\mathrm{QED}}-0.951304 \mathcal{P}_{\psi \psi(14)}^{\mathrm{QED}}-0.118477 \mathcal{P}_{\psi \psi(15)}^{\mathrm{QED}} \\
& -0.413930 \mathcal{P}_{\psi \psi(16)}^{\mathrm{QED}}+1.365234 \mathcal{P}_{\psi \psi(17)}^{\mathrm{QED}}-0.118477 \mathcal{P}_{\psi \psi(18)}^{\mathrm{QED}} \\
& -1.365234 \mathcal{P}_{\psi \psi(19)}^{\mathrm{QED}}+0.118477 \mathcal{P}_{\psi \psi(20)}^{\mathrm{QED}}+1.779164 \mathcal{P}_{\psi \psi(21)}^{\mathrm{QED}} \\
& +0.118477 \mathcal{P}_{\psi \psi(22)}^{\mathrm{QED}}-0.236954 \mathcal{P}_{\psi \psi(23)}^{\mathrm{QED}}+0.413930 \mathcal{P}_{\psi \psi(24)}^{\mathrm{QED}} \\
& -0.118477 \mathcal{P}_{\psi \psi(25)}^{\mathrm{QED}}-0.118477 \mathcal{P}_{\psi \psi(26)}^{\mathrm{QED}}-0.354640 \mathcal{P}_{\psi \psi(27)}^{\mathrm{QED}} \\
& -0.354640 \mathcal{P}_{\psi \psi(28)}^{\mathrm{QED}}-0.354640 \mathcal{P}_{\psi \psi(29)}^{\mathrm{QED}}-0.354640 \mathcal{P}_{\psi \psi(30)}^{\mathrm{QED}} \\
& -0.354640 \mathcal{P}_{\psi \psi(31)}^{\mathrm{QED}}-0.354640 \mathcal{P}_{\psi \psi(32)}^{\mathrm{QED}}-0.152021 \mathcal{P}_{\psi \psi(33)}^{\mathrm{QED}} \\
& +0.152021 \mathcal{P}_{\psi \psi(34)}^{\mathrm{QED}}-0.287628 \mathcal{P}_{\psi \psi(35)}^{\mathrm{QED}}-0.071907 \mathcal{P}_{\psi \psi(36)}^{\mathrm{QED}} \\
& +0.071907 \mathcal{P}_{\psi \psi(37)}^{\mathrm{QED}}+0.287628 \mathcal{P}_{\psi \psi(38)}^{\mathrm{QED}}+0.14381 \mathcal{P}_{\psi \psi(39)}^{\mathrm{QED}} \\
& -0.071907 \mathcal{P}_{\psi \psi(40)}^{\mathrm{QED}}+0.143814 \mathcal{P}_{\psi \psi(41)}^{\mathrm{QED}}-0.071907 \mathcal{P}_{\psi \psi(42)}^{\mathrm{QED}} \\
& -0.071907 \mathcal{P}_{\psi \psi(43)}^{\mathrm{QED}}+0.071907 \mathcal{P}_{\psi \psi(44)}^{\mathrm{QED}}-0.287628 \mathcal{P}_{\psi \psi(45)}^{\mathrm{QED}}
\end{aligned}
$$




$$
\begin{aligned}
& -0.143814 \mathcal{P}_{\psi \psi(46)}^{\mathrm{QED}}+0.287628 \mathcal{P}_{\psi \psi(47)}^{\mathrm{QED}}+0.071907 \mathcal{P}_{\psi \psi(48)}^{\mathrm{QED}} \\
& +0.071907 \mathcal{P}_{\psi \psi(49)}^{\mathrm{QED}}-0.143814 \mathcal{P}_{\psi \psi(50)}^{\mathrm{QED}}-0.314625 \mathcal{P}_{\psi \psi(51)}^{\mathrm{QED}} \\
& -0.075682 \mathcal{P}_{\psi \psi(52)}^{\mathrm{QED}}-0.238943 \mathcal{P}_{\psi \psi(53)}^{\mathrm{QED}}+0.477887 \mathcal{P}_{\psi \psi(54)}^{\mathrm{QED}} \\
& +0.238944 \mathcal{P}_{\psi \psi(55)}^{\mathrm{QED}}+0.238944 \mathcal{P}_{\psi \psi(56)}^{\mathrm{QED}}+0.238944 \mathcal{P}_{\psi \psi(57)}^{\mathrm{QED}} \\
& +0.238944 \mathcal{P}_{\psi \psi(58)}^{\mathrm{QED}}-0.075682 \mathcal{P}_{\psi \psi(59)}^{\mathrm{QED}}-0.238943 \mathcal{P}_{\psi \psi(60)}^{\mathrm{QED}} \\
& -0.287628 \mathcal{P}_{\psi \psi(61)}^{\mathrm{QED}}+0.287628 \mathcal{P}_{\psi \psi(62)}^{\mathrm{QED}}-0.314625 \mathcal{P}_{\psi \psi(63)}^{\mathrm{QED}} \\
& -0.238943 \mathcal{P}_{\psi \psi(64)}^{\mathrm{QED}}+0.314625 \mathcal{P}_{\psi \psi(65)}^{\mathrm{QED}}+0.075682 \mathcal{P}_{\psi \psi(66)}^{\mathrm{QED}} \\
& +0.075682 \mathcal{P}_{\psi \psi(67)}^{\mathrm{QED}}-0.238943 \mathcal{P}_{\psi \psi(68)}^{\mathrm{QED}}-0.477887 \mathcal{P}_{\psi \psi(69)}^{\mathrm{QED}} \\
& \left.+0.314625 \mathcal{P}_{\psi \psi(70)}^{\mathrm{QED}}-0.528590 \mathcal{P}_{\psi \psi(71)}^{\mathrm{QED}}+0.528590 \mathcal{P}_{\psi \psi(72)}^{\mathrm{QED}}\right] a \\
& +O\left(a^{2}\right)
\end{aligned}
$$

where alliances between tensor structures is again manifest. In contrast to (3.11) there are less than half the number of Lorentz structures.

\section{Discussion.}

We have completed the one loop evaluation of all the possible 4-point Green's functions in QCD in a linear covariant gauge at the completely symmetric subtraction point. The initial computations of [8, 9] concentrated on the quartic gluon function also at the fully symmetric point and this article completes the symmetric point programme. While the aim was partly to achieve this, the results should prove useful to Schwinger-Dyson studies of 4-point functions. The solutions to such equations should overlap with the perturbative information given here and can be used to ensure that the approximations used to truncate the tower of Green's function are in fact consistent with independent results. In turn such consistency should impinge upon the Schwinger-Dyson analyses of the 3-point functions of QCD where effects from 4-point kernels have been ignored in the first instance and models of swordfish diagram contributions are now being used. There are several directions in which the present work can be extended to further complement such Schwinger-Dyson analyses. The first is to go the next order in perturbation theory for all six 4-point functions. This is not a straightforward task as the two loop master integrals at the fully symmetric subtraction point, which would emerge from a REDUZE reduction, are not yet known. Once these are available then the next order can be completed. For the five Green's functions considered here this will involve a renormalization which in passing acts as a check on the one loop analysis. A second direction would be to repeat the one loop computation but at the fully off-shell point. While the one loop masters are already known from [35] the reduction of the necessary scalar Feynman integrals lurking within each Green's functions would need to be constructed first. Aside from these two tasks one immediate application of the formalism recorded here is to the study of QCD gauge fixing in gauges other than the linear covariant one. We have noted two such gauges which are the Curci-Ferrari one, [18, and the maximal abelian gauge, 36, 37, 38. Although these are nonlinear gauges they have been of interest due to their potential connection with colour confinement. Indeed the 3-point vertex functions of QCD have been studied in these other gauges using Schwinger-Dyson techniques in order to extract a point of view on gluon confinement which is consistent in different gauges and hence is of physical relevance. A side dish to such a programme is ensuring the consistent implementation of the Slavnov-Taylor identities in the running down to the infrared region. Some such identities 
involve several of the Green's functions evaluated here and so our analysis should prove useful for that programme.

Acknowledgements. The author thanks Prof. J. Pawlowski for discussions and the encouragement to carry out this work as well as Prof. A. Eichhorn and the ITP, University of Heidelberg for hospitality during the visit where it was initiated. The work was carried out with the support of the STFC through the Consolidated Grant ST/L000431/1.

\section{A Tensor bases.}

For reference we provide the Lorentz tensor bases for each of the three Green's functions where we used the projection method. The basis which emerged from the explicit one loop computation of the quartic quark Green's function is also included as well as the analogous basis for QED. On notation, where a generalized $\Gamma(n)$-matrix has a contraction with an external momentum we use the convention that the vector appears in place of the contracting index. First, the ten basis elements for the gluon-ghost function are

$$
\begin{aligned}
& \mathcal{P}_{A c(1)}^{\sigma \rho}=\eta^{\sigma \rho}, \quad \mathcal{P}_{A c(2)}^{\sigma \rho}=\frac{p^{\sigma} p^{\rho}}{\mu^{2}}, \quad \mathcal{P}_{A c(3)}^{\sigma \rho}=\frac{p^{\sigma} q^{\rho}}{\mu^{2}}, \mathcal{P}_{A c(4)}^{\sigma \rho}=\frac{p^{\sigma} r^{\rho}}{\mu^{2}} \\
& \mathcal{P}_{A c(5)}^{\sigma \rho}=\frac{q^{\sigma} p^{\rho}}{\mu^{2}}, \mathcal{P}_{A c(6)}^{\sigma \rho}=\frac{q^{\sigma} q^{\rho}}{\mu^{2}}, \mathcal{P}_{A c(7)}^{\sigma \rho}=\frac{q^{\sigma} r^{\rho}}{\mu^{2}}, \mathcal{P}_{A c(8)}^{\sigma \rho}=\frac{r^{\sigma} p^{\rho}}{\mu^{2}} \\
& \mathcal{P}_{A c(9)}^{\sigma \rho}=\frac{r^{\sigma} q^{\rho}}{\mu^{2}}, \mathcal{P}_{A c(10)}^{\sigma \rho}=\frac{r^{\sigma} r^{\rho}}{\mu^{2}} .
\end{aligned}
$$

We have included the mass scale $\mu$ to ensure that all the tensors have the same mass dimension. The colour group factor is absent in the Lorentz basis but appears explicitly in the results section. For the gluon-quark 4-point function there are 68 tensors but with the use of the $\Gamma_{(n)}$-matrices this partitions into subspaces of rank 36,31 and 1 . We have

$$
\begin{aligned}
& \mathcal{P}_{A \psi(1)}^{\sigma \rho}(p, q, r)=\gamma^{\sigma} p^{\rho}, \mathcal{P}_{A \psi(2)}^{\sigma \rho}(p, q, r)=\gamma^{\sigma} q^{\rho}, \mathcal{P}_{A \psi(3)}^{\sigma \rho}(p, q, r)=\gamma^{\sigma} r^{\rho} \\
& \mathcal{P}_{A \psi(4)}^{\sigma \rho}(p, q, r)=\gamma^{\rho} p^{\sigma}, \mathcal{P}_{A \psi(5)}^{\sigma \rho}(p, q, r)=\gamma^{\rho} q^{\sigma}, \mathcal{P}_{A \psi(6)}^{\sigma \rho}(p, q, r)=\gamma^{\rho} r^{\sigma} \\
& \mathcal{P}_{A \psi(7)}^{\sigma \rho}(p, q, r)=\not p \eta^{\sigma \rho}, \mathcal{P}_{A \psi(8)}^{\sigma \rho}(p, q, r)=\not q \eta^{\sigma \rho}, \mathcal{P}_{A \psi(9)}^{\sigma \rho}(p, q, r)=\not \eta^{\sigma \rho} \\
& \mathcal{P}_{A \psi(10)}^{\sigma \rho}(p, q, r)=\frac{\not p p^{\sigma} p^{\rho}}{\mu^{2}}, \mathcal{P}_{A \psi(11)}^{\sigma \rho}(p, q, r)=\frac{\not p p^{\sigma} q^{\rho}}{\mu^{2}}, \mathcal{P}_{A \psi(12)}^{\sigma \rho}(p, q, r)=\frac{\not p p^{\sigma} r^{\rho}}{\mu^{2}} \\
& \mathcal{P}_{A \psi(13)}^{\sigma \rho}(p, q, r)=\frac{\not q^{\sigma} p^{\rho}}{\mu^{2}}, \mathcal{P}_{A \psi(14)}^{\sigma \rho}(p, q, r)=\frac{\not p q^{\sigma} q^{\rho}}{\mu^{2}}, \mathcal{P}_{A \psi(15)}^{\sigma \rho}(p, q, r)=\frac{\not p q^{\sigma} r^{\rho}}{\mu^{2}} \\
& \mathcal{P}_{A \psi(16)}^{\sigma \rho}(p, q, r)=\frac{\not p r^{\sigma} p^{\rho}}{\mu^{2}}, \mathcal{P}_{A \psi(17)}^{\sigma \rho}(p, q, r)=\frac{\not p r^{\sigma} q^{\rho}}{\mu^{2}}, \mathcal{P}_{A \psi(18)}^{\sigma \rho}(p, q, r)=\frac{\not p r^{\sigma} r^{\rho}}{\mu^{2}} \\
& \mathcal{P}_{A \psi(19)}^{\sigma \rho}(p, q, r)=\frac{\not p^{\sigma} p^{\rho}}{\mu^{2}}, \mathcal{P}_{A \psi(20)}^{\sigma \rho}(p, q, r)=\frac{\not p^{\sigma} q^{\rho}}{\mu^{2}}, \mathcal{P}_{A \psi(21)}^{\sigma \rho}(p, q, r)=\frac{\not p^{\sigma} r^{\rho}}{\mu^{2}} \\
& \mathcal{P}_{A \psi(22)}^{\sigma \rho}(p, q, r)=\frac{\not q^{\sigma} p^{\rho}}{\mu^{2}}, \mathcal{P}_{A \psi(23)}^{\sigma \rho}(p, q, r)=\frac{\not q^{\sigma} q^{\rho}}{\mu^{2}}, \mathcal{P}_{A \psi(24)}^{\sigma \rho}(p, q, r)=\frac{\not q^{\sigma} r^{\rho}}{\mu^{2}} \\
& \mathcal{P}_{A \psi(25)}^{\sigma \rho}(p, q, r)=\frac{\not r^{\sigma} p^{\rho}}{\mu^{2}}, \mathcal{P}_{A \psi(26)}^{\sigma \rho}(p, q, r)=\frac{\not r^{\sigma} q^{\rho}}{\mu^{2}}, \mathcal{P}_{A \psi(27)}^{\sigma \rho}(p, q, r)=\frac{\not r^{\sigma} r^{\rho}}{\mu^{2}} \\
& \mathcal{P}_{A \psi(28)}^{\sigma \rho}(p, q, r)=\frac{\not p^{\sigma} p^{\rho}}{\mu^{2}}, \mathcal{P}_{A \psi(29)}^{\sigma \rho}(p, q, r)=\frac{\not p^{\sigma} q^{\rho}}{\mu^{2}}, \mathcal{P}_{A \psi(30)}^{\sigma \rho}(p, q, r)=\frac{\not p^{\sigma} r^{\rho}}{\mu^{2}}
\end{aligned}
$$




$$
\begin{aligned}
& \mathcal{P}_{A \psi(31)}^{\sigma \rho}(p, q, r)=\frac{\not q^{\sigma} p^{\rho}}{\mu^{2}}, \mathcal{P}_{A \psi(32)}^{\sigma \rho}(p, q, r)=\frac{\not q^{\sigma} q^{\rho}}{\mu^{2}}, \quad \mathcal{P}_{A \psi(33)}^{\sigma \rho}(p, q, r)=\frac{\not q^{\sigma} r^{\rho}}{\mu^{2}} \\
& \mathcal{P}_{A \psi(34)}^{\sigma \rho}(p, q, r)=\frac{\not r^{\sigma} p^{\rho}}{\mu^{2}}, \mathcal{P}_{A \psi(35)}^{\sigma \rho}(p, q, r)=\frac{\not r^{\sigma} q^{\rho}}{\mu^{2}}, \mathcal{P}_{A \psi(36)}^{\sigma \rho}(p, q, r)=\frac{\not r^{\sigma} r^{\rho}}{\mu^{2}} \\
& \mathcal{P}_{A \psi(37)}^{\sigma \rho}(p, q, r)=\Gamma_{(3)}^{\sigma \rho p}, \mathcal{P}_{A \psi(38)}^{\sigma \rho}(p, q, r)=\Gamma_{(3)}^{\sigma \rho q}, \mathcal{P}_{A \psi(39)}^{\sigma \rho}(p, q, r)=\Gamma_{(3)}^{\sigma \rho r} \\
& \mathcal{P}_{A \psi(40)}^{\sigma \rho}(p, q, r)=\frac{\Gamma_{(3)}^{\sigma p q} p^{\rho}}{\mu^{2}}, \mathcal{P}_{A \psi(41)}^{\sigma \rho}(p, q, r)=\frac{\Gamma_{(3)}^{\sigma p q} q^{\rho}}{\mu^{2}}, \mathcal{P}_{A \psi(42)}^{\sigma \rho}(p, q, r)=\frac{\Gamma_{(3)}^{\sigma p q} r^{\rho}}{\mu^{2}} \\
& \mathcal{P}_{A \psi(43)}^{\sigma \rho}(p, q, r)=\frac{\Gamma_{(3)}^{\sigma q r} p^{\rho}}{\mu^{2}}, \mathcal{P}_{A \psi(44)}^{\sigma \rho}(p, q, r)=\frac{\Gamma_{(3)}^{\sigma q r} q^{\rho}}{\mu^{2}}, \quad \mathcal{P}_{A \psi(45)}^{\sigma \rho}(p, q, r)=\frac{\Gamma_{(3)}^{\sigma q r} r^{\rho}}{\mu^{2}} \\
& \mathcal{P}_{A \psi(46)}^{\sigma \rho}(p, q, r)=\frac{\Gamma_{(3)}^{\sigma r p} p^{\rho}}{\mu^{2}}, \mathcal{P}_{A \psi(47)}^{\sigma \rho}(p, q, r)=\frac{\Gamma_{(3)}^{\sigma r p} q^{\rho}}{\mu^{2}}, \mathcal{P}_{A \psi(48)}^{\sigma \rho}(p, q, r)=\frac{\Gamma_{(3)}^{\sigma r p} r^{\rho}}{\mu^{2}} \\
& \mathcal{P}_{A \psi(49)}^{\sigma \rho}(p, q, r)=\frac{\Gamma_{(3)}^{\rho p q} p^{\sigma}}{\mu^{2}}, \mathcal{P}_{A \psi(50)}^{\sigma \rho}(p, q, r)=\frac{\Gamma_{(3)}^{\rho p q} q^{\sigma}}{\mu^{2}}, \mathcal{P}_{A \psi(51)}^{\sigma \rho}(p, q, r)=\frac{\Gamma_{(3)}^{\rho p q} r^{\sigma}}{\mu^{2}} \\
& \mathcal{P}_{A \psi(52)}^{\sigma \rho}(p, q, r)=\frac{\Gamma_{(3)}^{\rho q r} p^{\sigma}}{\mu^{2}}, \mathcal{P}_{A \psi(53)}^{\sigma \rho}(p, q, r)=\frac{\Gamma_{(3)}^{\rho q r} q^{\sigma}}{\mu^{2}}, \mathcal{P}_{A \psi(54)}^{\sigma \rho}(p, q, r)=\frac{\Gamma_{(3)}^{\rho q r} r^{\sigma}}{\mu^{2}} \\
& \mathcal{P}_{A \psi(55)}^{\sigma \rho}(p, q, r)=\frac{\Gamma_{(3)}^{\rho r p} p^{\sigma}}{\mu^{2}}, \mathcal{P}_{A \psi(56)}^{\sigma \rho}(p, q, r)=\frac{\Gamma_{(3)}^{\rho r p} q^{\sigma}}{\mu^{2}}, \mathcal{P}_{A \psi(57)}^{\sigma \rho}(p, q, r)=\frac{\Gamma_{(3)}^{\rho r p} r^{\sigma}}{\mu^{2}} \\
& \mathcal{P}_{A \psi(58)}^{\sigma \rho}(p, q, r)=\frac{\Gamma_{(3)}^{p q r} \eta^{\sigma \rho}}{\mu^{2}}, \quad \mathcal{P}_{A \psi(59)}^{\sigma \rho}(p, q, r)=\frac{\Gamma_{(3)}^{p q r} p^{\sigma} p^{\rho}}{\mu^{4}}, \quad \mathcal{P}_{A \psi(60)}^{\sigma \rho}(p, q, r)=\frac{\Gamma_{(3)}^{p q r} p^{\sigma} q^{\rho}}{\mu^{4}} \\
& \mathcal{P}_{A \psi(61)}^{\sigma \rho}(p, q, r)=\frac{\Gamma_{(3)}^{p q r} p^{\sigma} r^{\rho}}{\mu^{4}}, \mathcal{P}_{A \psi(62)}^{\sigma \rho}(p, q, r)=\frac{\Gamma_{(3)}^{p q r} q^{\sigma} p^{\rho}}{\mu^{4}}, \mathcal{P}_{A \psi(63)}^{\sigma \rho}(p, q, r)=\frac{\Gamma_{(3)}^{p q r} q^{\sigma} q^{\rho}}{\mu^{4}} \\
& \mathcal{P}_{A \psi(64)}^{\sigma \rho}(p, q, r)=\frac{\Gamma_{(3)}^{p q r} q^{\sigma} r^{\rho}}{\mu^{4}}, \mathcal{P}_{A \psi(65)}^{\sigma \rho}(p, q, r)=\frac{\Gamma_{(3)}^{p q r} r^{\sigma} p^{\rho}}{\mu^{4}}, \mathcal{P}_{A \psi(66)}^{\sigma \rho}(p, q, r)=\frac{\Gamma_{(3)}^{p q r} r^{\sigma} q^{\rho}}{\mu^{4}} \\
& \mathcal{P}_{A \psi(67)}^{\sigma \rho}(p, q, r)=\frac{\Gamma_{(3)}^{p q r} r^{\sigma} r^{\rho}}{\mu^{4}}, \mathcal{P}_{A \psi(68)}^{\sigma \rho}(p, q, r)=\frac{\Gamma_{(5)}^{\sigma \rho p q r}}{\mu^{2}} .
\end{aligned}
$$

In the data file the projection matrix for this basis is presented in block partition form. The elements of the $\Gamma_{(3)}$ partition there are numbered from 1 to 31 and that for $\Gamma_{(5)}$ is merely 1. To map to the labels for each sector to the full $68 \times 68$ projection matrix, for which (A.2) is the basis, 36 needs to be added to the labels of the $\Gamma_{(3)}$ partition and 67 to that for the $\Gamma_{(5)}$ partition in order to produce all the non-zero entries in $\mathcal{M}_{A \psi k k^{\prime}}$ where $1 \leq k \leq 68$. By contrast the basis for the ghost-quark 4-point function is somewhat smaller as

$$
\mathcal{P}_{c \psi(1)}=\not p, \mathcal{P}_{c \psi(2)}=\not q, \mathcal{P}_{c \psi(3)}=\not, \mathcal{P}_{c \psi(4)}=\frac{\Gamma_{(3)}^{p q r}}{\mu^{2}} .
$$

It is worth noting that the basis involving external quark legs would be larger if there was a non-zero quark mass.

As noted earlier the situation with the structure of the one loop quartic quark 4-point function is different. We have not endeavoured to construct the most general basis of Lorentz tensors due to the separate spinor strings. Instead we provide the tensors which emerged as a consequence of the direct one loop computation to make contact with the electronic format of the data file. These are

$$
\begin{aligned}
& \mathcal{P}_{\psi \psi(1)}=\Gamma_{(1) \alpha}^{p}{ }_{\alpha}^{\beta} \Gamma_{(1) \delta}^{p}{ }^{\gamma} \delta^{i j} \delta^{k l} \delta_{I J} \delta_{K L} \quad, \quad \mathcal{P}_{\psi \psi(2)}=\Gamma_{(1) \alpha}^{p}{ }_{\alpha}^{\beta} \Gamma_{(1) \delta}^{p}{ }^{\gamma} \delta^{i j} \delta^{k l} \delta_{I K} \delta_{J L}
\end{aligned}
$$

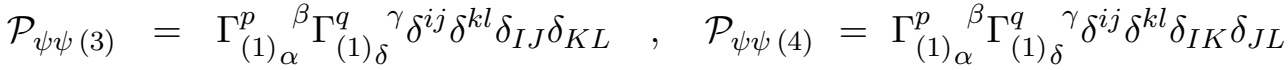




$$
\begin{aligned}
& \mathcal{P}_{\psi \psi(5)}=\Gamma_{(1) \alpha}^{p}{ }^{\beta} \Gamma_{(1)_{\delta}}^{\gamma} \delta^{i j} \delta^{k l} \delta_{I J} \delta_{K L} \quad, \quad \mathcal{P}_{\psi \psi(6)}=\Gamma_{(1) \alpha}^{p}{ }^{\beta} \Gamma_{(1)}^{r}{ }_{\delta}^{\gamma} \delta^{i j} \delta^{k l} \delta_{I K} \delta_{J L}
\end{aligned}
$$

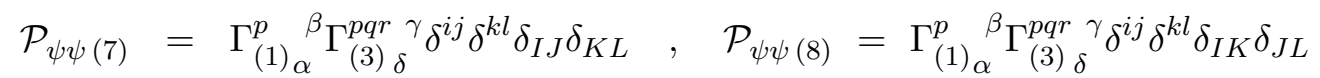

$$
\begin{aligned}
& \mathcal{P}_{\psi \psi(9)}=\Gamma_{(1) \alpha}^{p}{ }_{\alpha}^{\gamma} \Gamma_{(1) \delta}^{p}{ }^{\beta} \delta^{i k} \delta^{j l} \delta_{I J} \delta_{K L} \quad, \quad \mathcal{P}_{\psi \psi(10)}=\Gamma_{(1) \alpha}^{p}{ }_{\alpha}^{\gamma} \Gamma_{(1) \delta}^{p}{ }^{\beta} \delta^{i k} \delta^{j l} \delta_{I K} \delta_{J L} \\
& \mathcal{P}_{\psi \psi(11)}=\Gamma_{(1) \alpha}^{p}{ }^{\gamma} \Gamma_{(1) \delta}^{q}{ }^{\beta} \delta^{i k} \delta^{j l} \delta_{I J} \delta_{K L} \quad, \quad \mathcal{P}_{\psi \psi(12)}=\Gamma_{(1) \alpha}^{p}{ }^{\gamma} \Gamma_{(1) \delta}^{q}{ }^{\beta} \delta^{i k} \delta^{j l} \delta_{I K} \delta_{J L} \\
& \mathcal{P}_{\psi \psi(13)}=\Gamma_{(1) \alpha}^{p}{ }^{\gamma} \Gamma_{(1){ }_{\delta}}^{r} \delta^{i k} \delta^{j l} \delta_{I J} \delta_{K L} \quad, \quad \mathcal{P}_{\psi \psi(14)}=\Gamma_{(1) \alpha}^{p}{ }^{\gamma} \Gamma_{(1){ }_{\delta}}^{r} \delta^{i k} \delta^{j l} \delta_{I K} \delta_{J L} \\
& \mathcal{P}_{\psi \psi(15)}=\Gamma_{(1) \alpha}^{p} \Gamma_{(3) \delta}^{p q r} \delta^{i k} \delta^{j l} \delta_{I J} \delta_{K L} \quad, \quad \mathcal{P}_{\psi \psi(16)}=\Gamma_{(1) \alpha}^{p}{ }^{\gamma} \Gamma_{(3) \delta}^{p q r} \delta^{i k} \delta^{j l} \delta_{I K} \delta_{J L} \\
& \mathcal{P}_{\psi \psi(17)}=\Gamma_{(1) \delta}^{p}{ }^{\beta} \Gamma_{(1) \alpha}^{q}{ }_{\alpha}^{\gamma} \delta^{i k} \delta^{j l} \delta_{I J} \delta_{K L} \quad, \quad \mathcal{P}_{\psi \psi(18)}=\Gamma_{(1){ }_{\delta}}^{p} \Gamma_{(1)}^{q}{ }_{\alpha}^{\gamma} \delta^{i k} \delta^{j l} \delta_{I K} \delta_{J L} \\
& \mathcal{P}_{\psi \psi(19)}=\Gamma_{(1){ }_{\delta}}^{p} \Gamma_{(1) \alpha}^{r}{ }_{\alpha}^{\gamma} \delta^{i k} \delta^{j l} \delta_{I J} \delta_{K L} \quad, \quad \mathcal{P}_{\psi \psi(20)}=\Gamma_{(1){ }_{\delta}}^{p} \Gamma_{(1)}^{r}{ }_{\alpha}^{\gamma} \delta^{i k} \delta^{j l} \delta_{I K} \delta_{J L} \\
& \mathcal{P}_{\psi \psi(21)}=\Gamma_{(1) \delta}^{p}{ }^{\beta} \Gamma_{(3) \alpha}^{p q r} \gamma_{\alpha} \delta^{i k} \delta^{j l} \delta_{I J} \delta_{K L} \quad, \quad \mathcal{P}_{\psi \psi(22)}=\Gamma_{(1) \delta}^{p}{ }^{\beta} \Gamma_{(3) \alpha}^{p q r}{ }_{\alpha} \delta^{i k} \delta^{j l} \delta_{I K} \delta_{J L} \\
& \mathcal{P}_{\psi \psi(23)}=\Gamma_{(1){ }_{\delta}}^{p} \Gamma_{(1) \alpha}^{q}{ }_{\alpha}^{\beta} \delta^{i j} \delta^{k l} \delta_{I J} \delta_{K L} \quad, \quad \mathcal{P}_{\psi \psi(24)}=\Gamma_{(1){ }_{\delta}}^{p} \Gamma_{(1) \alpha}^{q}{ }_{\alpha}^{\beta} \delta^{i j} \delta^{k l} \delta_{I K} \delta_{J L} \\
& \mathcal{P}_{\psi \psi(25)}=\Gamma_{(1) \delta_{\delta}}^{p} \Gamma_{(1) \alpha}^{r}{ }_{\alpha}^{\beta} \delta^{i j} \delta^{k l} \delta_{I J} \delta_{K L} \quad, \quad \mathcal{P}_{\psi \psi(26)}=\Gamma_{(1){ }_{\delta}}^{p} \Gamma_{(1) \alpha}^{r}{ }_{\alpha}^{\beta} \delta^{i j} \delta^{k l} \delta_{I K} \delta_{J L} \\
& \mathcal{P}_{\psi \psi(27)}=\Gamma_{(1)_{\delta}}^{p} \Gamma_{(3)_{\alpha}{ }^{\beta} \delta^{i j} \delta^{k l} \delta_{I J} \delta_{K L}}^{p q r}, \quad \mathcal{P}_{\psi \psi(28)}=\Gamma_{(1)_{\delta}}^{p} \Gamma_{(3) \alpha}^{p q r \beta} \delta^{i j} \delta^{k l} \delta_{I K} \delta_{J L} \\
& \mathcal{P}_{\psi \psi(29)}=\Gamma_{(1) \alpha}^{q}{ }^{\beta} \Gamma_{(1) \delta_{\delta}}^{q} \delta^{i j} \delta^{k l} \delta_{I J} \delta_{K L} \quad, \quad \mathcal{P}_{\psi \psi(30)}=\Gamma_{(1) \alpha}^{q}{ }^{\beta} \Gamma_{(1) \delta}^{q}{ }^{\gamma} \delta^{i j} \delta^{k l} \delta_{I K} \delta_{J L} \\
& \mathcal{P}_{\psi \psi(31)}=\Gamma_{(1) \alpha}^{q}{ }_{\alpha}^{\beta} \Gamma_{(1) \delta}^{r}{ }_{\delta}^{\gamma} \delta^{i j} \delta^{k l} \delta_{I J} \delta_{K L} \quad, \quad \mathcal{P}_{\psi \psi(32)}=\Gamma_{(1) \alpha}^{q}{ }_{\alpha}^{\beta} \Gamma_{(1) \delta}^{r}{ }^{\gamma} \delta^{i j} \delta^{k l} \delta_{I K} \delta_{J L} \\
& \mathcal{P}_{\psi \psi(33)}=\Gamma_{(1) \alpha}^{q}{ }_{\alpha}^{\beta} \Gamma_{(3)_{\delta}}^{p q r} \delta^{i j} \delta^{k l} \delta_{I J} \delta_{K L} \quad, \quad \mathcal{P}_{\psi \psi(34)}=\Gamma_{(1) \alpha}^{q}{ }_{\alpha}^{\beta} \Gamma_{(3)_{\delta}}^{p q r} \delta^{i j} \delta^{k l} \delta_{I K} \delta_{J L} \\
& \mathcal{P}_{\psi \psi(35)}=\Gamma_{(1) \alpha}^{q}{ }^{\gamma} \Gamma_{(1) \delta}^{q}{ }^{\beta} \delta^{i k} \delta^{j l} \delta_{I J} \delta_{K L} \quad, \quad \mathcal{P}_{\psi \psi(36)}=\Gamma_{(1) \alpha}^{q}{ }^{\gamma} \Gamma_{(1) \delta}^{q}{ }_{j}^{\beta} \delta^{i k} \delta^{j l} \delta_{I K} \delta_{J L} \\
& \mathcal{P}_{\psi \psi(37)}=\Gamma_{(1) \alpha}^{q}{ }^{\gamma} \Gamma_{(1) \delta}^{r}{ }^{\beta} \delta^{i k} \delta^{j l} \delta_{I J} \delta_{K L} \quad, \quad \mathcal{P}_{\psi \psi(38)}=\Gamma_{(1) \alpha}^{q}{ }^{\gamma} \Gamma_{(1) \delta}^{r}{ }^{\beta}{ }^{i k} \delta^{j l} \delta_{I K} \delta_{J L} \\
& \mathcal{P}_{\psi \psi(39)}=\Gamma_{(1) \alpha}^{q}{ }_{\alpha}^{\gamma} \Gamma_{(3) \delta}^{p q r} \delta^{\beta} \delta^{i k} \delta^{j l} \delta_{I J} \delta_{K L} \quad, \quad \mathcal{P}_{\psi \psi(40)}=\Gamma_{(1) \alpha}^{q}{ }_{\alpha}^{\gamma} \Gamma_{(3) \delta}^{p q r}{ }_{\delta} \delta^{i k} \delta^{j l} \delta_{I K} \delta_{J L} \\
& \mathcal{P}_{\psi \psi(41)}=\Gamma_{(1){ }_{\delta}}^{q} \Gamma_{(1) \alpha}^{r}{ }_{\alpha}^{\gamma} \delta^{i k} \delta^{j l} \delta_{I J} \delta_{K L} \quad, \quad \mathcal{P}_{\psi \psi(42)}=\Gamma_{(1){ }_{\delta}}^{q} \Gamma_{(1) \alpha}^{r}{ }_{\alpha}^{\gamma} \delta^{i k} \delta^{j l} \delta_{I K} \delta_{J L}
\end{aligned}
$$

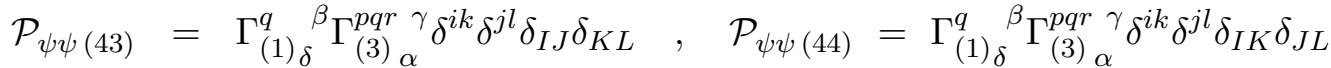

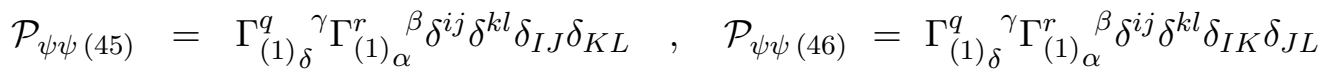

$$
\begin{aligned}
& \mathcal{P}_{\psi \psi(47)}=\Gamma_{(1){ }_{\delta}}^{q} \Gamma_{(3) \alpha}^{p q r} \delta^{i j} \delta^{k l} \delta_{I J} \delta_{K L} \quad, \quad \mathcal{P}_{\psi \psi(48)}=\Gamma_{(1){ }_{\delta}}^{q} \Gamma_{(3) \alpha}^{p q r \beta} \delta^{i j} \delta^{k l} \delta_{I K} \delta_{J L} \\
& \mathcal{P}_{\psi \psi(49)}=\Gamma_{(1) \alpha}^{r}{ }_{\alpha}^{\beta} \Gamma_{(1)}^{r}{ }_{\delta}^{\gamma} \delta^{i j} \delta^{k l} \delta_{I J} \delta_{K L} \quad, \quad \mathcal{P}_{\psi \psi(50)}=\Gamma_{(1) \alpha}^{r}{ }^{\beta} \Gamma_{(1)}^{r}{ }_{\delta}^{\gamma} \delta^{i j} \delta^{k l} \delta_{I K} \delta_{J L} \\
& \mathcal{P}_{\psi \psi(51)}=\Gamma_{(1) \alpha}^{r} \Gamma_{(3) \delta}^{p q r} \delta^{i j} \delta^{k l} \delta_{I J} \delta_{K L} \quad, \quad \mathcal{P}_{\psi \psi(52)}=\Gamma_{(1) \alpha}^{r} \Gamma_{(3) \delta}^{p q r} \delta^{i j} \delta^{k l} \delta_{I K} \delta_{J L} \\
& \mathcal{P}_{\psi \psi(53)}=\Gamma_{(1) \alpha}^{r}{ }_{\alpha}^{\gamma} \Gamma_{(1){ }_{\delta}}^{r} \delta^{i k} \delta^{j l} \delta_{I J} \delta_{K L} \quad, \quad \mathcal{P}_{\psi \psi(54)}=\Gamma_{(1) \alpha}^{r}{ }^{\gamma} \Gamma_{(1) \delta}^{r}{ }_{\delta}^{\beta}{ }^{i k} \delta^{j l} \delta_{I K} \delta_{J L} \\
& \mathcal{P}_{\psi \psi(55)}=\Gamma_{(1) \alpha}^{r}{ }_{\alpha}^{\gamma} \Gamma_{(3) \delta}^{p q r} \delta^{i k} \delta^{j l} \delta_{I J} \delta_{K L} \quad, \quad \mathcal{P}_{\psi \psi(56)}=\Gamma_{(1) \alpha}^{r}{ }^{\gamma} \Gamma_{(3) \delta}^{p q r} \delta^{i k} \delta^{j l} \delta_{I K} \delta_{J L} \\
& \mathcal{P}_{\psi \psi(57)}=\Gamma_{(1) \delta}^{r}{ }_{\delta}^{\beta} \Gamma_{(3) \alpha}^{p q r}{ }_{\alpha} \delta^{i k} \delta^{j l} \delta_{I J} \delta_{K L} \quad, \quad \mathcal{P}_{\psi \psi(58)}=\Gamma_{(1) \delta_{\delta}}^{\beta} \Gamma_{(3) \alpha}^{p q r}{ }^{i k} \delta^{j l} \delta_{I K} \delta_{J L} \\
& \mathcal{P}_{\psi \psi(59)}=\Gamma_{(1) \delta}^{r}{ }_{\delta}^{\gamma} \Gamma_{(3) \alpha}^{p q r}{ }_{\alpha} \delta^{i j} \delta^{k l} \delta_{I J} \delta_{K L} \quad, \quad \mathcal{P}_{\psi \psi(60)}=\Gamma_{(1) \delta}^{r}{ }^{\gamma} \Gamma_{(3) \alpha}^{p q r}{ }_{\alpha} \delta^{i j} \delta^{k l} \delta_{I K} \delta_{J L} \\
& \mathcal{P}_{\psi \psi(61)}=\Gamma_{(1)_{\alpha}}^{\mu} \Gamma_{(1)_{\delta}}^{\mu} \delta^{i j} \delta^{k l} \delta_{I J} \delta_{K L} \quad, \quad \mathcal{P}_{\psi \psi(62)}=\Gamma_{(1)_{\alpha}}^{\mu{ }^{\beta}} \Gamma_{(1) \delta}^{\mu}{ }^{\gamma} \delta^{i j} \delta^{k l} \delta_{I K} \delta_{J L}
\end{aligned}
$$

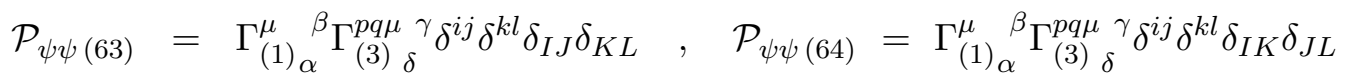

$$
\begin{aligned}
& \mathcal{P}_{\psi \psi(65)}=\Gamma_{(1) \alpha}^{\mu}{ }_{\alpha}^{\beta} \Gamma_{(3) \delta}^{p r \mu \gamma} \delta^{i j} \delta^{k l} \delta_{I J} \delta_{K L} \quad, \quad \mathcal{P}_{\psi \psi(66)}=\Gamma_{(1) \alpha}^{\mu}{ }_{\alpha}^{\beta} \Gamma_{(3) \delta}^{p r \mu \gamma} \delta^{i j} \delta^{k l} \delta_{I K} \delta_{J L} \\
& \mathcal{P}_{\psi \psi(67)}=\Gamma_{(1) \alpha}^{\mu}{ }_{\alpha}^{\beta} \Gamma_{(3) \delta}^{q r \mu \gamma} \delta^{i j} \delta^{k l} \delta_{I J} \delta_{K L} \quad, \quad \mathcal{P}_{\psi \psi(68)}=\Gamma_{(1) \alpha}^{\mu}{ }_{\alpha}^{\beta} \Gamma_{(3) \delta}^{q r \mu \gamma} \delta^{i j} \delta^{k l} \delta_{I K} \delta_{J L}
\end{aligned}
$$




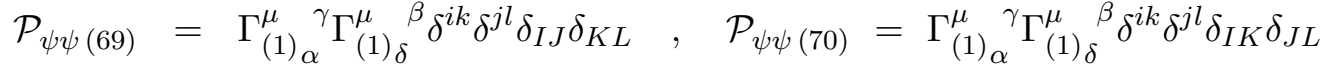

$$
\begin{aligned}
& \mathcal{P}_{\psi \psi(71)}=\Gamma_{(1) \alpha}^{\mu}{ }_{\alpha}^{\gamma} \Gamma_{(3) \delta}^{p q \mu \beta} \delta^{i k} \delta^{j l} \delta_{I J} \delta_{K L} \quad, \quad \mathcal{P}_{\psi \psi(72)}=\Gamma_{(1) \alpha}^{\mu}{ }_{\alpha}^{\gamma} \Gamma_{(3) \delta}^{p q \mu \beta} \delta^{i k} \delta^{j l} \delta_{I K} \delta_{J L} \\
& \mathcal{P}_{\psi \psi(73)}=\Gamma_{(1) \alpha}^{\mu}{ }_{\alpha}^{\gamma} \Gamma_{(3) \delta}^{p r \mu \beta} \delta^{i k} \delta^{j l} \delta_{I J} \delta_{K L} \quad, \quad \mathcal{P}_{\psi \psi(74)}=\Gamma_{(1) \alpha}^{\mu}{ }_{\alpha}^{\gamma} \Gamma_{(3) \delta}^{p r \mu \beta} \delta^{i k} \delta^{j l} \delta_{I K} \delta_{J L} \\
& \mathcal{P}_{\psi \psi(75)}=\Gamma_{(1) \alpha}^{\mu}{ }_{\alpha}^{\gamma} \Gamma_{(3) \delta}^{q r \mu \beta} \delta^{i k} \delta^{j l} \delta_{I J} \delta_{K L} \quad, \quad \mathcal{P}_{\psi \psi(76)}=\Gamma_{(1) \alpha}^{\mu}{ }_{\alpha}^{\gamma} \Gamma_{(3) \delta}^{q r \mu \beta} \delta^{i k} \delta^{j l} \delta_{I K} \delta_{J L} \\
& \mathcal{P}_{\psi \psi(77)}=\Gamma_{(1){ }_{\delta}}^{\mu} \Gamma_{(3) \alpha}^{p q \mu \gamma} \delta^{i k} \delta^{j l} \delta_{I J} \delta_{K L} \quad, \quad \mathcal{P}_{\psi \psi(78)}=\Gamma_{(1){ }_{\delta}}^{\mu} \Gamma_{(3)}^{p q \mu}{ }_{\alpha} \delta^{i k} \delta^{j l} \delta_{I K} \delta_{J L} \\
& \mathcal{P}_{\psi \psi(79)}=\Gamma_{(1) \delta}^{\mu}{ }_{\delta}^{\beta} \Gamma_{(3) \alpha}^{p r \mu \gamma} \delta^{i k} \delta^{j l} \delta_{I J} \delta_{K L} \quad, \quad \mathcal{P}_{\psi \psi(80)}=\Gamma_{(1)_{\delta}}^{\mu} \Gamma_{(3)}^{p r \mu \gamma} \delta^{i k} \delta^{j l} \delta_{I K} \delta_{J L} \\
& \mathcal{P}_{\psi \psi(81)}=\Gamma_{(1) \delta}^{\mu}{ }^{\beta} \Gamma_{(3) \alpha}^{q r \mu \gamma} \delta^{i k} \delta^{j l} \delta_{I J} \delta_{K L} \quad, \quad \mathcal{P}_{\psi \psi(82)}=\Gamma_{(1) \delta}^{\mu}{ }_{j}^{\beta} \Gamma_{(3) \alpha}^{q r \mu \gamma} \delta^{i k} \delta^{j l} \delta_{I K} \delta_{J L} \\
& \mathcal{P}_{\psi \psi(83)}=\Gamma_{(1) \delta}^{\mu}{ }_{\delta}^{\gamma} \Gamma_{(3) \alpha}^{p q \mu \beta} \delta^{i j} \delta^{k l} \delta_{I J} \delta_{K L} \quad, \quad \mathcal{P}_{\psi \psi(84)}=\Gamma_{(1) \delta_{\delta}}^{\mu} \Gamma_{(3) \alpha}^{p q \mu \beta} \delta^{i j} \delta^{k l} \delta_{I K} \delta_{J L} \\
& \mathcal{P}_{\psi \psi(85)}=\Gamma_{(1) \delta}^{\mu}{ }_{\delta}^{\gamma} \Gamma_{(3) \alpha}^{p r \mu \beta} \delta^{i j} \delta^{k l} \delta_{I J} \delta_{K L} \quad, \quad \mathcal{P}_{\psi \psi(86)}=\Gamma_{(1) \delta}^{\mu}{ }^{\gamma} \Gamma_{(3) \alpha}^{p r \mu \beta} \delta^{i j} \delta^{k l} \delta_{I K} \delta_{J L} \\
& \mathcal{P}_{\psi \psi(87)}=\Gamma_{(1) \delta}^{\mu}{ }^{\gamma} \Gamma_{(3) \alpha}^{q r \mu \beta} \delta^{i j} \delta^{k l} \delta_{I J} \delta_{K L} \quad, \quad \mathcal{P}_{\psi \psi(88)}=\Gamma_{(1) \delta}^{\mu}{ }^{\gamma} \Gamma_{(3) \alpha}^{q r \mu \beta} \delta^{i j} \delta^{k l} \delta_{I K} \delta_{J L} \\
& \mathcal{P}_{\psi \psi(89)}=\Gamma_{(3) \alpha}^{p q r} \Gamma_{(3) \delta}^{p q r} \delta^{i j} \delta^{k l} \delta_{I J} \delta_{K L} \quad, \quad \mathcal{P}_{\psi \psi(90)}=\Gamma_{(3) \alpha}^{p q r} \Gamma_{(3) \delta}^{p q r} \delta^{i j} \delta^{k l} \delta_{I K} \delta_{J L} \\
& \mathcal{P}_{\psi \psi(91)}=\Gamma_{(3) \alpha}^{p q r} \Gamma_{(3) \delta}^{p q r} \delta^{i k} \delta^{j l} \delta_{I J} \delta_{K L} \quad, \quad \mathcal{P}_{\psi \psi(92)}=\Gamma_{(3) \alpha}^{p q r} \Gamma_{(3) \delta}^{p q r} \delta^{i k} \delta^{j l} \delta_{I K} \delta_{J L} \\
& \mathcal{P}_{\psi \psi(93)}=\Gamma_{(3) \alpha}^{p q \sigma \beta} \Gamma_{(3) \delta}^{p q \sigma \gamma} \delta^{i j} \delta^{k l} \delta_{I J} \delta_{K L} \quad, \quad \mathcal{P}_{\psi \psi(94)}=\Gamma_{(3) \alpha}^{p q \sigma} \Gamma_{(3) \delta}^{p q \sigma \gamma} \delta^{i j} \delta^{k l} \delta_{I K} \delta_{J L} \\
& \mathcal{P}_{\psi \psi(95)}=\Gamma_{(3) \alpha}^{p q \sigma \beta} \Gamma_{(3) \delta}^{p r \sigma \gamma} \delta^{i j} \delta^{k l} \delta_{I J} \delta_{K L} \quad, \quad \mathcal{P}_{\psi \psi(96)}=\Gamma_{(3) \alpha}^{p q \sigma \beta} \Gamma_{(3) \delta}^{p r \sigma \gamma} \delta^{i j} \delta^{k l} \delta_{I K} \delta_{J L} \\
& \mathcal{P}_{\psi \psi(97)}=\Gamma_{(3) \alpha}^{p q \sigma \beta} \Gamma_{(3) \delta}^{q r \sigma \gamma} \delta^{i j} \delta^{k l} \delta_{I J} \delta_{K L} \quad, \quad \mathcal{P}_{\psi \psi(98)}=\Gamma_{(3) \alpha}^{p q \sigma \beta} \Gamma_{(3) \delta}^{q r \sigma \gamma} \delta^{i j} \delta^{k l} \delta_{I K} \delta_{J L} \\
& \mathcal{P}_{\psi \psi(99)}=\Gamma_{(3) \alpha}^{p q \sigma} \Gamma_{(3) \delta}^{p q \sigma \beta} \delta^{i k} \delta^{j l} \delta_{I J} \delta_{K L} \quad, \quad \mathcal{P}_{\psi \psi(100)}=\Gamma_{(3) \alpha}^{p q \sigma} \Gamma_{(3) \delta}^{p q \sigma \beta} \delta^{i k} \delta^{j l} \delta_{I K} \delta_{J L} \\
& \mathcal{P}_{\psi \psi(101)}=\Gamma_{(3) \alpha}^{p q \sigma \gamma} \Gamma_{(3) \delta}^{p r \sigma \beta} \delta^{i k} \delta^{j l} \delta_{I J} \delta_{K L} \quad, \quad \mathcal{P}_{\psi \psi(102)}=\Gamma_{(3) \alpha}^{p q \sigma}{ }_{\alpha} \Gamma_{(3) \delta}^{p r \sigma \beta} \delta^{i k} \delta^{j l} \delta_{I K} \delta_{J L} \\
& \mathcal{P}_{\psi \psi(103)}=\Gamma_{(3) \alpha}^{p q \sigma}{ }_{\alpha} \Gamma_{(3) \delta}^{q r \sigma \beta} \delta^{i k} \delta^{j l} \delta_{I J} \delta_{K L} \quad, \quad \mathcal{P}_{\psi \psi(104)}=\Gamma_{(3){ }_{\alpha}{ }_{(3) \delta}^{p q \sigma}{ }^{q}{ }^{i k} \delta^{j l} \delta_{I K} \delta_{J L}}^{q r \sigma} \\
& \mathcal{P}_{\psi \psi(105)}=\Gamma_{(3) \delta}^{p q \sigma \beta} \Gamma_{(3) \alpha}^{p r \sigma \gamma} \delta^{i k} \delta^{j l} \delta_{I J} \delta_{K L} \quad, \quad \mathcal{P}_{\psi \psi(106)}=\Gamma_{(3) \delta}^{p q \sigma \beta} \Gamma_{(3) \alpha}^{p r \sigma \gamma} \delta^{i k} \delta^{j l} \delta_{I K} \delta_{J L} \\
& \mathcal{P}_{\psi \psi(107)}=\Gamma_{(3) \delta}^{p q \sigma \beta} \Gamma_{(3) \alpha}^{q r \sigma \gamma} \delta^{i k} \delta^{j l} \delta_{I J} \delta_{K L} \quad, \quad \mathcal{P}_{\psi \psi(108)}=\Gamma_{(3) \delta}^{p q \sigma \beta} \Gamma_{(3) \alpha}^{q r \sigma}{ }_{\alpha} \delta^{i k} \delta^{j l} \delta_{I K} \delta_{J L} \\
& \mathcal{P}_{\psi \psi(109)}=\Gamma_{(3) \delta}^{p q \sigma \gamma} \Gamma_{(3) \alpha}^{p r \sigma \beta} \delta^{i j} \delta^{k l} \delta_{I J} \delta_{K L} \quad, \quad \mathcal{P}_{\psi \psi(110)}=\Gamma_{(3) \delta}^{p q \sigma}{ }_{\delta} \Gamma_{(3) \alpha}^{p r \sigma \beta} \delta^{i j} \delta^{k l} \delta_{I K} \delta_{J L} \\
& \mathcal{P}_{\psi \psi(111)}=\Gamma_{(3) \delta}^{p q \sigma \gamma} \Gamma_{(3) \alpha}^{q r \sigma \beta} \delta^{i j} \delta^{k l} \delta_{I J} \delta_{K L} \quad, \quad \mathcal{P}_{\psi \psi(112)}=\Gamma_{(3) \delta}^{p q \sigma}{ }_{\delta}^{\gamma} \Gamma_{(3) \alpha}^{q r \sigma \beta} \delta^{i j} \delta^{k l} \delta_{I K} \delta_{J L} \\
& \mathcal{P}_{\psi \psi(113)}=\Gamma_{(3) \alpha}^{p r \sigma \beta} \Gamma_{(3) \delta}^{p r \sigma \gamma} \delta^{i j} \delta^{k l} \delta_{I J} \delta_{K L} \quad, \quad \mathcal{P}_{\psi \psi(114)}=\Gamma_{(3) \alpha}^{p r \sigma \beta} \Gamma_{(3) \delta}^{p r \sigma \gamma} \delta^{i j} \delta^{k l} \delta_{I K} \delta_{J L} \\
& \mathcal{P}_{\psi \psi(115)}=\Gamma_{(3) \alpha}^{p r \sigma \beta} \Gamma_{(3) \delta}^{q r \sigma \gamma} \delta^{i j} \delta^{k l} \delta_{I J} \delta_{K L} \quad, \quad \mathcal{P}_{\psi \psi(116)}=\Gamma_{(3) \alpha}^{p r \sigma} \Gamma_{(3) \delta}^{q r \sigma}{ }_{\delta} \delta^{i j} \delta^{k l} \delta_{I K} \delta_{J L} \\
& \mathcal{P}_{\psi \psi(117)}=\Gamma_{(3) \alpha}^{p r \sigma \gamma} \Gamma_{(3) \delta}^{p r \sigma \beta} \delta^{i k} \delta^{j l} \delta_{I J} \delta_{K L} \quad, \quad \mathcal{P}_{\psi \psi(118)}=\Gamma_{(3) \alpha}^{p r \sigma \gamma} \Gamma_{(3) \delta}^{p r \sigma \beta} \delta^{i k} \delta^{j l} \delta_{I K} \delta_{J L} \\
& \mathcal{P}_{\psi \psi(119)}=\Gamma_{(3) \alpha}^{p r \sigma \gamma} \Gamma_{(3) \delta}^{q r \sigma \beta} \delta^{i k} \delta^{j l} \delta_{I J} \delta_{K L} \quad, \quad \mathcal{P}_{\psi \psi(120)}=\Gamma_{(3) \alpha}^{p r \sigma \gamma} \Gamma_{(3) \delta}^{q r \sigma \beta} \delta^{i k} \delta^{j l} \delta_{I K} \delta_{J L} \\
& \mathcal{P}_{\psi \psi(121)}=\Gamma_{(3) \delta}^{p r \sigma \beta} \Gamma_{(3) \alpha}^{q r \sigma \gamma} \delta^{i k} \delta^{j l} \delta_{I J} \delta_{K L} \quad, \quad \mathcal{P}_{\psi \psi(122)}=\Gamma_{(3) \delta}^{p r \sigma \beta} \Gamma_{(3) \alpha}^{q r \sigma}{ }_{\alpha} \delta^{i k} \delta^{j l} \delta_{I K} \delta_{J L} \\
& \mathcal{P}_{\psi \psi(123)}=\Gamma_{(3) \delta}^{p r \sigma \gamma} \Gamma_{(3) \alpha}^{q r \sigma \beta} \delta^{i j} \delta^{k l} \delta_{I J} \delta_{K L} \quad, \quad \mathcal{P}_{\psi \psi(124)}=\Gamma_{(3){ }_{\delta}}^{p r \sigma} \Gamma_{(3) \alpha}^{q r \sigma \beta} \delta^{i j} \delta^{k l} \delta_{I K} \delta_{J L} \\
& \mathcal{P}_{\psi \psi(125)}=\Gamma_{(3) \alpha}^{p \nu \sigma \beta} \Gamma_{(3) \delta}^{p \nu \sigma \gamma} \delta^{i j} \delta^{k l} \delta_{I J} \delta_{K L} \quad, \quad \mathcal{P}_{\psi \psi(126)}=\Gamma_{(3) \alpha}^{p \nu \sigma \beta} \Gamma_{(3) \delta}^{p \nu \sigma \gamma} \delta^{i j} \delta^{k l} \delta_{I K} \delta_{J L} \\
& \mathcal{P}_{\psi \psi(127)}=\Gamma_{(3) \alpha}^{p \nu \sigma \beta} \Gamma_{(3) \delta}^{q \nu \sigma \gamma} \delta^{i j} \delta^{k l} \delta_{I J} \delta_{K L} \quad, \quad \mathcal{P}_{\psi \psi(128)}=\Gamma_{(3) \alpha}^{p \nu \sigma \beta} \Gamma_{(3) \delta}^{q \nu \sigma \gamma} \delta^{i j} \delta^{k l} \delta_{I K} \delta_{J L} \\
& \mathcal{P}_{\psi \psi(129)}=\Gamma_{(3) \alpha}^{p \nu \sigma \beta} \Gamma_{(3) \delta}^{r \nu \sigma \gamma} \delta^{i j} \delta^{k l} \delta_{I J} \delta_{K L} \quad, \quad \mathcal{P}_{\psi \psi(130)}=\Gamma_{(3){ }_{\alpha}{ }^{\beta} \Gamma_{(3) \delta}^{r \nu \sigma \gamma} \delta^{i j} \delta^{k l} \delta_{I K} \delta_{J L}}^{p \nu \sigma} \\
& \mathcal{P}_{\psi \psi(131)}=\Gamma_{(3) \alpha}^{p \nu \sigma \gamma} \Gamma_{(3) \delta}^{p \nu \sigma \beta} \delta^{i k} \delta^{j l} \delta_{I J} \delta_{K L} \quad, \quad \mathcal{P}_{\psi \psi(132)}=\Gamma_{(3) \alpha}^{p \nu \sigma \gamma} \Gamma_{(3) \delta}^{p \nu \sigma \beta} \delta^{i k} \delta^{j l} \delta_{I K} \delta_{J L}
\end{aligned}
$$




$$
\begin{aligned}
& \mathcal{P}_{\psi \psi(133)}=\Gamma_{(3) \alpha \alpha}^{p \nu \sigma \gamma} \Gamma_{(3) \delta}^{q \nu \sigma \beta} \delta^{i k} \delta^{j l} \delta_{I J} \delta_{K L} \quad, \quad \mathcal{P}_{\psi \psi(134)}=\Gamma_{(3) \alpha}^{p \nu \sigma} \Gamma_{(3) \delta}^{q \nu \sigma \beta} \delta^{i k} \delta^{j l} \delta_{I K} \delta_{J L} \\
& \mathcal{P}_{\psi \psi(135)}=\Gamma_{(3) \alpha}^{p \nu \sigma \gamma} \Gamma_{(3) \delta}^{r \nu \sigma \beta} \delta^{i k} \delta^{j l} \delta_{I J} \delta_{K L} \quad, \quad \mathcal{P}_{\psi \psi(136)}=\Gamma_{(3){ }_{\alpha}}^{p \nu \sigma} \Gamma_{(3) \delta}^{r \nu \sigma \beta} \delta^{i k} \delta^{j l} \delta_{I K} \delta_{J L} \\
& \mathcal{P}_{\psi \psi(137)}=\Gamma_{(3) \delta}^{p \nu \sigma \beta} \Gamma_{(3) \alpha}^{q \nu \sigma \gamma} \delta^{i k} \delta^{j l} \delta_{I J} \delta_{K L} \quad, \quad \mathcal{P}_{\psi \psi(138)}=\Gamma_{(3) \delta}^{p \nu \sigma \beta} \Gamma_{(3) \alpha}^{q \nu \sigma \gamma} \delta^{i k} \delta^{j l} \delta_{I K} \delta_{J L} \\
& \mathcal{P}_{\psi \psi(139)}=\Gamma_{(3) \delta}^{p \nu \sigma \beta} \Gamma_{(3) \alpha}^{r \nu \sigma \gamma} \delta^{i k} \delta^{j l} \delta_{I J} \delta_{K L} \quad, \quad \mathcal{P}_{\psi \psi(140)}=\Gamma_{(3) \delta}^{p \nu \sigma \beta} \Gamma_{(3) \alpha}^{r \nu \sigma \gamma} \delta^{i k} \delta^{j l} \delta_{I K} \delta_{J L} \\
& \mathcal{P}_{\psi \psi(141)}=\Gamma_{(3) \delta}^{p \nu \sigma \gamma} \Gamma_{(3) \alpha}^{q \nu \sigma \beta} \delta^{i j} \delta^{k l} \delta_{I J} \delta_{K L} \quad, \quad \mathcal{P}_{\psi \psi(142)}=\Gamma_{(3) \delta}^{p \nu \sigma \gamma} \Gamma_{(3) \alpha}^{q \nu \sigma \beta} \delta^{i j} \delta^{k l} \delta_{I K} \delta_{J L} \\
& \mathcal{P}_{\psi \psi(143)}=\Gamma_{(3) \delta}^{p \nu \sigma \gamma} \Gamma_{(3) \alpha}^{r \nu \sigma \beta} \delta^{i j} \delta^{k l} \delta_{I J} \delta_{K L} \quad, \quad \mathcal{P}_{\psi \psi(144)}=\Gamma_{(3) \delta}^{p \nu \sigma \gamma} \Gamma_{(3) \alpha}^{r \nu \sigma \beta} \delta^{i j} \delta^{k l} \delta_{I K} \delta_{J L} \\
& \mathcal{P}_{\psi \psi(145)}=\Gamma_{(3) \alpha}^{q r \sigma \beta} \Gamma_{(3) \delta}^{q r \sigma \gamma} \delta^{i j} \delta^{k l} \delta_{I J} \delta_{K L} \quad, \quad \mathcal{P}_{\psi \psi(146)}=\Gamma_{(3) \alpha}^{q r \sigma \beta} \Gamma_{(3) \delta}^{q r \sigma \gamma} \delta^{i j} \delta^{k l} \delta_{I K} \delta_{J L} \\
& \mathcal{P}_{\psi \psi(147)}=\Gamma_{(3) \alpha}^{q r \sigma}{ }_{\alpha}^{\gamma} \Gamma_{(3) \delta}^{q r \sigma \beta} \delta^{i k} \delta^{j l} \delta_{I J} \delta_{K L} \quad, \quad \mathcal{P}_{\psi \psi(148)}=\Gamma_{(3) \alpha}^{q r \sigma}{ }_{\alpha} \Gamma_{(3) \delta}^{q r \sigma \beta} \delta^{i k} \delta^{j l} \delta_{I K} \delta_{J L} \\
& \mathcal{P}_{\psi \psi(149)}=\Gamma_{(3) \alpha}^{q \nu \sigma \beta} \Gamma_{(3) \delta}^{q \nu \sigma \gamma} \delta^{i j} \delta^{k l} \delta_{I J} \delta_{K L} \quad, \quad \mathcal{P}_{\psi \psi(150)}=\Gamma_{(3) \alpha}^{q \nu \sigma \beta} \Gamma_{(3) \delta}^{q \nu \sigma \gamma} \delta^{i j} \delta^{k l} \delta_{I K} \delta_{J L} \\
& \mathcal{P}_{\psi \psi(151)}=\Gamma_{(3) \alpha}^{q \nu \sigma \beta} \Gamma_{(3) \delta}^{r \nu \sigma \gamma} \delta^{i j} \delta^{k l} \delta_{I J} \delta_{K L} \quad, \quad \mathcal{P}_{\psi \psi(152)}=\Gamma_{(3) \alpha}^{q \nu \sigma \beta} \Gamma_{(3) \delta}^{r \nu \sigma \gamma} \delta^{i j} \delta^{k l} \delta_{I K} \delta_{J L} \\
& \mathcal{P}_{\psi \psi(153)}=\Gamma_{(3) \alpha}^{q \nu \sigma \gamma} \Gamma_{(3) \delta}^{q \nu \sigma \beta} \delta^{i k} \delta^{j l} \delta_{I J} \delta_{K L} \quad, \quad \mathcal{P}_{\psi \psi(154)}=\Gamma_{(3) \alpha}^{q \nu \sigma \gamma} \Gamma_{(3) \delta}^{q \nu \sigma \beta} \delta^{i k} \delta^{j l} \delta_{I K} \delta_{J L}
\end{aligned}
$$

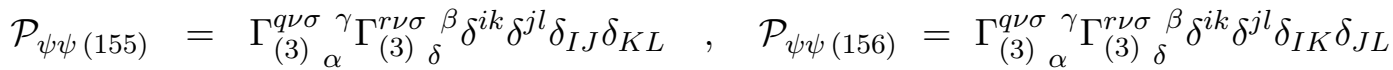

$$
\begin{aligned}
& \mathcal{P}_{\psi \psi(157)}=\Gamma_{(3) \delta}^{q \nu \sigma \beta} \Gamma_{(3) \alpha}^{r \nu \sigma \gamma} \delta^{i k} \delta^{j l} \delta_{I J} \delta_{K L} \quad, \quad \mathcal{P}_{\psi \psi(158)}=\Gamma_{(3){ }_{\delta}}^{q \nu \sigma \beta} \Gamma_{(3) \alpha}^{r \nu \sigma \gamma} \delta^{i k} \delta^{j l} \delta_{I K} \delta_{J L} \\
& \mathcal{P}_{\psi \psi(159)}=\Gamma_{(3) \delta}^{q \nu \sigma \gamma} \Gamma_{(3) \alpha}^{r \nu \sigma \beta} \delta^{i j} \delta^{k l} \delta_{I J} \delta_{K L} \quad, \quad \mathcal{P}_{\psi \psi(160)}=\Gamma_{(3) \delta}^{q \nu \sigma \gamma} \Gamma_{(3) \alpha}^{r \nu \sigma \beta} \delta^{i j} \delta^{k l} \delta_{I K} \delta_{J L} \\
& \mathcal{P}_{\psi \psi(161)}=\Gamma_{(3) \alpha \alpha}^{r \nu \sigma \beta} \Gamma_{(3) \delta}^{r \nu \sigma \gamma} \delta^{i j} \delta^{k l} \delta_{I J} \delta_{K L} \quad, \quad \mathcal{P}_{\psi \psi(162)}=\Gamma_{(3){ }_{\alpha}{ }^{\beta} \Gamma_{(3) \delta}^{r \nu \sigma \gamma} \delta^{i j} \delta^{k l} \delta_{I K} \delta_{J L}} \\
& \mathcal{P}_{\psi \psi(163)}=\Gamma_{(3) \alpha}^{r \nu \sigma \gamma} \Gamma_{(3) \delta}^{r \nu \sigma \beta} \delta^{i k} \delta^{j l} \delta_{I J} \delta_{K L} \quad, \quad \mathcal{P}_{\psi \psi(164)}=\Gamma_{(3) \alpha}^{r \nu \sigma} \Gamma_{(3) \delta}^{r \nu \sigma \beta} \delta^{i k} \delta^{j l} \delta_{I K} \delta_{J L} \\
& \mathcal{P}_{\psi \psi(165)}=\Gamma_{(3) \alpha}^{\mu \nu \sigma \beta} \Gamma_{(3) \delta}^{\mu \nu \sigma \gamma} \delta^{i j} \delta^{k l} \delta_{I J} \delta_{K L} \quad, \quad \mathcal{P}_{\psi \psi(166)}=\Gamma_{(3){ }_{\alpha}{ }^{\beta} \Gamma_{(3) \delta}^{\mu \nu \sigma \gamma} \delta^{i j} \delta^{k l} \delta_{I K} \delta_{J L}}
\end{aligned}
$$

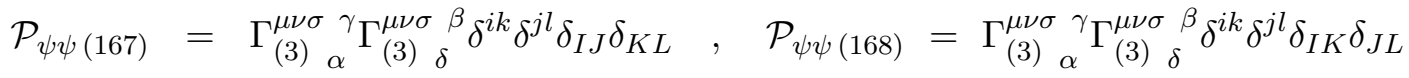

where we have suppressed the mass scale $\mu$ for presentational reasons. It can be restored by using a dimensional argument. When higher loop corrections are included in this Green's function we expect the basis to be larger. For instance tensors such as $\Gamma_{(5)}^{\mu \nu p q r} \otimes \Gamma_{(5)}^{\mu \nu p q r}$ should appear at two loops given the number of $\gamma$-matrices which would arise.

By contrast the basis for the quartic electron Green's function in QED is a smaller basis than that for the quark. This is partly due to the absence of the colour group theory but also due to a smaller number of contributing Feynman graphs. We have

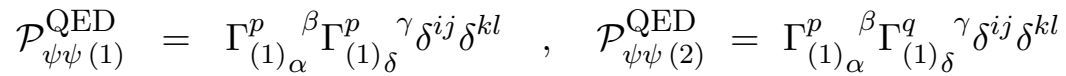

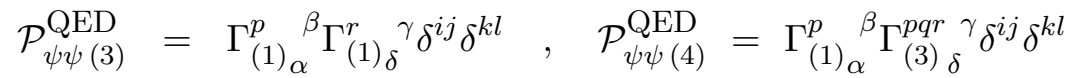

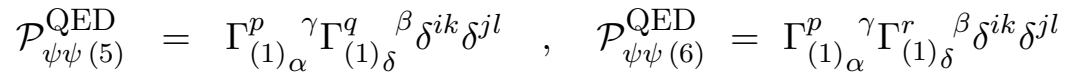

$$
\begin{aligned}
& \mathcal{P}_{\psi \psi(7)}^{\mathrm{QED}}=\Gamma_{(1)_{\alpha}}^{p} \Gamma_{(3) \delta}^{p q r} \delta^{i k} \delta^{j l} \quad, \quad \mathcal{P}_{\psi \psi(8)}^{\mathrm{QED}}=\Gamma_{(1) \delta}^{p}{ }^{\beta} \Gamma_{(1)_{\alpha}{ }^{\gamma} \delta^{i k} \delta^{j l}}
\end{aligned}
$$

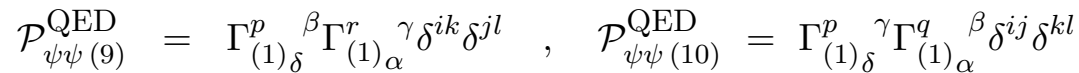

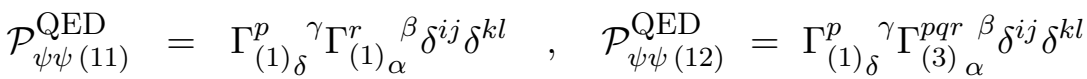

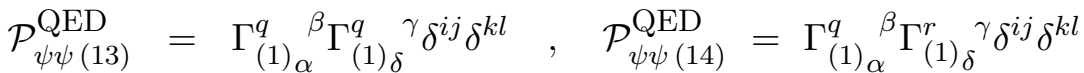

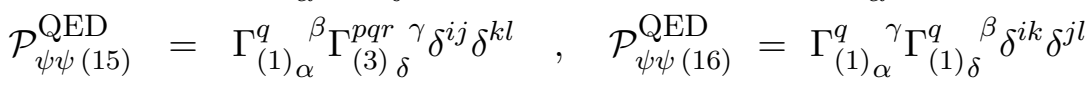

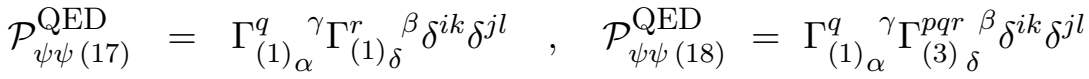




$$
\begin{aligned}
& \mathcal{P}_{\psi \psi(19)}^{\mathrm{QED}}=\Gamma_{(1) \delta}^{q}{ }^{\beta} \Gamma_{(1) \alpha}^{r}{ }_{\alpha}^{\gamma} \delta^{i k} \delta^{j l} \quad, \quad \mathcal{P}_{\psi \psi(20)}^{\mathrm{QED}}=\Gamma_{(1) \delta}^{q}{ }^{\beta} \Gamma_{(3) \alpha}^{p q r}{ }_{\alpha} \delta^{i k} \delta^{j l} \\
& \mathcal{P}_{\psi \psi(21)}^{\mathrm{QED}}=\Gamma_{(1)_{\delta}}^{q} \Gamma_{(1) \alpha}^{r} \delta^{i j} \delta^{k l} \quad, \quad \mathcal{P}_{\psi \psi(22)}^{\mathrm{QED}}=\Gamma_{(1)_{\delta}}^{q} \Gamma_{(3) \alpha}^{p q r} \delta^{i j} \delta^{k l} \\
& \mathcal{P}_{\psi \psi(23)}^{\mathrm{QED}}=\Gamma_{(1) \alpha}^{r} \Gamma_{(3) \delta}^{p q r}{ }^{\gamma} \delta^{i j} \delta^{k l} \quad, \quad \mathcal{P}_{\psi \psi(24)}^{\mathrm{QED}}=\Gamma_{(1) \alpha}^{r}{ }_{\alpha}^{\gamma} \Gamma_{(1) \delta_{\delta}{ }^{\beta} \delta^{i k} \delta^{j l}}
\end{aligned}
$$

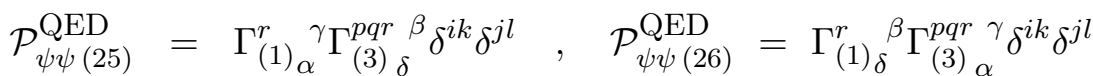

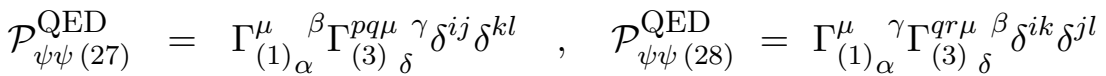

$$
\begin{aligned}
& \mathcal{P}_{\psi \psi(29)}^{\mathrm{QED}}=\Gamma_{(1) \delta}^{\mu{ }^{\beta}} \Gamma_{(3) \alpha}^{p q \mu \gamma} \delta^{i k} \delta^{j l} \quad, \quad \mathcal{P}_{\psi \psi(30)}^{\mathrm{QED}}=\Gamma_{(1) \delta}^{\mu{ }^{\beta}} \Gamma_{(3) \alpha}^{p r \mu \gamma} \delta^{i k} \delta^{j l}
\end{aligned}
$$

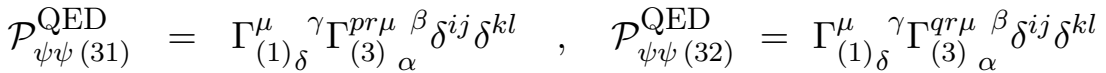

$$
\begin{aligned}
& \mathcal{P}_{\psi \psi(33)}^{\mathrm{QED}}=\Gamma_{(3) \alpha}^{p q r} \Gamma_{(3) \delta}^{p q r} \delta^{i j} \delta^{k l} \quad, \quad \mathcal{P}_{\psi \psi(34)}^{\mathrm{QED}}=\Gamma_{(3) \alpha}^{p q r}{ }_{(3) \delta}^{p q r} \delta_{(3)}^{i k} \delta^{j l} \\
& \mathcal{P}_{\psi \psi(35)}^{\mathrm{QED}}=\Gamma_{(3) \alpha}^{p q \sigma \beta} \Gamma_{(3) \delta}^{p q \sigma \gamma} \delta^{i j} \delta^{k l} \quad, \quad \mathcal{P}_{\psi \psi(36)}^{\mathrm{QED}}=\Gamma_{(3) \alpha}^{p q \sigma \beta} \Gamma_{(3) \delta}^{p r \sigma \gamma} \delta^{i j} \delta^{k l} \\
& \mathcal{P}_{\psi \psi(37)}^{\mathrm{QED}}=\Gamma_{(3) \alpha}^{p q \sigma \beta} \Gamma_{(3) \delta}^{q r \sigma \gamma} \delta^{i j} \delta^{k l} \quad, \quad \mathcal{P}_{\psi \psi(38)}^{\mathrm{QED}}=\Gamma_{(3) \alpha}^{p q \sigma \gamma} \Gamma_{(3) \delta}^{p q \sigma \beta} \delta^{i k} \delta^{j l} \\
& \mathcal{P}_{\psi \psi(39)}^{\mathrm{QED}}=\Gamma_{(3) \alpha}^{p q \sigma} \Gamma_{(3) \delta}^{p r \sigma \beta} \delta^{i k} \delta^{j l} \quad, \quad \mathcal{P}_{\psi \psi(40)}^{\mathrm{QED}}=\Gamma_{(3) \alpha}^{p q \sigma}{ }_{\alpha} \Gamma_{(3) \delta}^{q r \sigma \beta} \delta^{i k} \delta^{j l} \\
& \mathcal{P}_{\psi \psi(41)}^{\mathrm{QED}}=\Gamma_{(3) \delta}^{p q \sigma \beta} \Gamma_{(3) \alpha}^{p r \sigma \gamma} \delta^{i k} \delta^{j l} \quad, \quad \mathcal{P}_{\psi \psi(42)}^{\mathrm{QED}}=\Gamma_{(3) \delta}^{p q \sigma \beta} \Gamma_{(3) \alpha}^{q r \sigma \gamma} \delta^{i k} \delta^{j l} \\
& \mathcal{P}_{\psi \psi(43)}^{\mathrm{QED}}=\Gamma_{(3) \delta}^{p q \sigma \gamma} \Gamma_{(3) \alpha}^{p r \sigma \beta} \delta^{i j} \delta^{k l} \quad, \quad \mathcal{P}_{\psi \psi(44)}^{\mathrm{QED}}=\Gamma_{(3) \delta}^{p q \sigma \gamma} \Gamma_{(3) \alpha}^{q r \sigma \beta} \delta^{i j} \delta^{k l} \\
& \mathcal{P}_{\psi \psi(45)}^{\mathrm{QED}}=\Gamma_{(3) \alpha}^{p r \sigma \beta} \Gamma_{(3) \delta}^{p r \sigma \gamma} \delta^{i j} \delta^{k l} \quad, \quad \mathcal{P}_{\psi \psi(46)}^{\mathrm{QED}}=\Gamma_{(3){ }_{\alpha}}^{p r \sigma \beta} \Gamma_{(3) \delta}^{q r \sigma} \delta^{i j} \delta^{k l} \\
& \mathcal{P}_{\psi \psi(47)}^{\mathrm{QED}}=\Gamma_{(3) \alpha}^{p r \sigma \gamma} \Gamma_{(3) \delta}^{p r \sigma \beta} \delta^{i k} \delta^{j l} \quad, \quad \mathcal{P}_{\psi \psi(48)}^{\mathrm{QED}}=\Gamma_{(3) \alpha}^{p r \sigma \gamma} \Gamma_{(3) \delta}^{q r \sigma \beta} \delta^{i k} \delta^{j l} \\
& \mathcal{P}_{\psi \psi(49)}^{\mathrm{QED}}=\Gamma_{(3) \delta}^{p r \sigma \beta} \Gamma_{(3) \alpha}^{q r \sigma \gamma} \delta^{i k} \delta^{j l} \quad, \quad \mathcal{P}_{\psi \psi(50)}^{\mathrm{QED}}=\Gamma_{(3) \delta}^{p r \sigma \gamma} \Gamma_{(3) \alpha}^{q r \sigma \beta} \delta^{i j} \delta^{k l} \\
& \mathcal{P}_{\psi \psi(51)}^{\mathrm{QED}}=\Gamma_{(3) \alpha}^{p \nu \sigma \beta} \Gamma_{(3) \delta}^{p \nu \sigma \gamma} \delta^{i j} \delta^{k l} \quad, \quad \mathcal{P}_{\psi \psi(52)}^{\mathrm{QED}}=\Gamma_{(3) \alpha}^{p \nu \sigma \beta} \Gamma_{(3) \delta}^{q \nu \sigma \gamma} \delta^{i j} \delta^{k l} \\
& \mathcal{P}_{\psi \psi(53)}^{\mathrm{QED}}=\Gamma_{(3) \alpha}^{p \nu \sigma \beta} \Gamma_{(3) \delta}^{r \nu \sigma \gamma} \delta^{i j} \delta^{k l} \quad, \quad \mathcal{P}_{\psi \psi(54)}^{\mathrm{QED}}=\Gamma_{(3) \alpha}^{p \nu \sigma \gamma} \Gamma_{(3) \delta}^{p \nu \sigma \beta} \delta^{i k} \delta^{j l} \\
& \mathcal{P}_{\psi \psi(55)}^{\mathrm{QED}}=\Gamma_{(3) \alpha}^{p \nu \sigma \gamma} \Gamma_{(3) \delta}^{q \nu \sigma \beta} \delta^{i k} \delta^{j l} \quad, \quad \mathcal{P}_{\psi \psi(56)}^{\mathrm{QED}}=\Gamma_{(3) \alpha}^{p \nu \sigma} \Gamma_{(3) \delta}^{r \nu \sigma \beta} \delta^{i k} \delta^{j l} \\
& \mathcal{P}_{\psi \psi(57)}^{\mathrm{QED}}=\Gamma_{(3) \delta}^{p \nu \sigma \beta} \Gamma_{(3) \alpha}^{q \nu \sigma \gamma} \delta^{i k} \delta^{j l} \quad, \quad \mathcal{P}_{\psi \psi(58)}^{\mathrm{QED}}=\Gamma_{(3) \delta}^{p \nu \sigma \beta} \Gamma_{(3) \alpha}^{r \nu \sigma \gamma} \delta^{i k} \delta^{j l} \\
& \mathcal{P}_{\psi \psi(59)}^{\mathrm{QED}}=\Gamma_{(3) \delta}^{p \nu \sigma \gamma} \Gamma_{(3) \alpha}^{q \nu \sigma \beta} \delta^{i j} \delta^{k l} \quad, \quad \mathcal{P}_{\psi \psi(60)}^{\mathrm{QED}}=\Gamma_{(3) \delta}^{p \nu \sigma \gamma} \Gamma_{(3) \alpha}^{r \nu \sigma \beta} \delta^{i j} \delta^{k l} \\
& \mathcal{P}_{\psi \psi(61)}^{\mathrm{QED}}=\Gamma_{(3) \alpha}^{q r \sigma \beta} \Gamma_{(3) \delta}^{q r \sigma \gamma} \delta^{i j} \delta^{k l} \quad, \quad \mathcal{P}_{\psi \psi(62)}^{\mathrm{QED}}=\Gamma_{(3) \alpha}^{q r \sigma \gamma} \Gamma_{(3) \delta}^{q r \sigma \beta} \delta^{i k} \delta^{j l} \\
& \mathcal{P}_{\psi \psi(63)}^{\mathrm{QED}}=\Gamma_{(3) \alpha}^{q \nu \sigma \beta} \Gamma_{(3) \delta}^{q \nu \sigma \gamma} \delta^{i j} \delta^{k l} \quad, \quad \mathcal{P}_{\psi \psi(64)}^{\mathrm{QED}}=\Gamma_{(3) \alpha}^{q \nu \sigma \beta} \Gamma_{(3) \delta}^{r \nu \sigma \gamma} \delta^{i j} \delta^{k l} \\
& \mathcal{P}_{\psi \psi(65)}^{\mathrm{QED}}=\Gamma_{(3) \alpha}^{q \nu \sigma \gamma} \Gamma_{(3) \delta}^{q \nu \sigma \beta} \delta^{i k} \delta^{j l} \quad, \quad \mathcal{P}_{\psi \psi(66)}^{\mathrm{QED}}=\Gamma_{(3) \alpha}^{q \nu \sigma \gamma} \Gamma_{(3) \delta}^{r \nu \sigma \beta} \delta^{i k} \delta^{j l} \\
& \mathcal{P}_{\psi \psi(67)}^{\mathrm{QED}}=\Gamma_{(3) \delta}^{q \nu \sigma \beta} \Gamma_{(3) \alpha}^{r \nu \sigma \gamma} \delta^{i k} \delta^{j l} \quad, \quad \mathcal{P}_{\psi \psi(68)}^{\mathrm{QED}}=\Gamma_{(3) \delta}^{q \nu \sigma \gamma} \Gamma_{(3) \alpha}^{r \nu \sigma \beta} \delta^{i j} \delta^{k l} \\
& \mathcal{P}_{\psi \psi(69)}^{\mathrm{QED}}=\Gamma_{(3) \alpha}^{r \nu \sigma} \Gamma_{(3) \delta}^{r \nu \sigma \gamma} \delta^{i j} \delta^{k l} \quad, \quad \mathcal{P}_{\psi \psi(70)}^{\mathrm{QED}}=\Gamma_{(3){ }_{\alpha} \Gamma_{(3) \delta}^{r \nu \sigma} \delta^{i k} \delta^{j l}}^{r \nu \sigma} \\
& \mathcal{P}_{\psi \psi(71)}^{\mathrm{QED}}=\Gamma_{(3) \alpha}^{\mu \nu \sigma \beta} \Gamma_{(3)_{\delta} \gamma}^{\mu \nu \sigma \gamma} \delta^{i j} \delta^{k l}, \quad \mathcal{P}_{\psi \psi(72)}^{\mathrm{QED}}=\Gamma_{(3) \alpha}^{\mu \nu \sigma \gamma} \Gamma_{(3) \delta}^{\mu \nu \sigma \beta} \delta^{i k} \delta^{j l} .
\end{aligned}
$$

Finally, we give the projection matrix for the ghost-quark 4-point function as an aid to orient with the information presented in the associated data file. It is the most compact of the 
projections we constructed. We have

$$
\mathcal{M}_{c \psi}=\frac{1}{64}\left(\begin{array}{cccc}
-24 & -12 & -12 & 0 \\
-12 & -24 & -12 & 0 \\
-12 & -12 & -24 & 0 \\
0 & 0 & 0 & 27
\end{array}\right) .
$$

The entries in this projection matrix are numbers in contrast with the others we constructed

which involve rational polynomials in $d$. This is because there are no Lorentz indices on the elements of the ghost-gluon Lorentz tensor basis.

\section{References.}

[1] D.J. Gross \& F.J. Wilczek, Phys. Rev. Lett. 30 (1973), 1343.

[2] H.D. Politzer, Phys. Rev. Lett. 30 (1973), 1346.

[3] P.A. Baikov, K.G. Chetyrkin \& J.H. Kühn, Phys. Rev. Lett. 118 (2017), 082002.

[4] F. Herzog, B. Ruijl, T. Ueda, J.A.M. Vermaseren \& A. Vogt, JHEP 1702 (2017), 090.

[5] A.L. Blum, M.Q. Huber, M. Mitter \& L. von Smekal, Phys. Rev. D89 (2014), 061703.

[6] A.L. Blum, R. Alkofer, M.Q. Huber \& A. Windisch, arXiv:1611.04827 [hep-ph].

[7] M. Mitter, J.M. Pawlowski \& N. Strodthoff, Phys. Rev. D91 (2015), 054035.

[8] P. Pascual \& R. Tarrach, Nucl. Phys. B174 (1980), 123; Nucl. Phys. B181 (1981), 546.

[9] J.A. Gracey, Phys. Rev. D90 (2014), 025011.

[10] C. Kellermann \& C.S. Fischer, Phys. Rev. D78 (2008), 025015.

[11] C. Kellermann \& C.S. Fischer, arXiv:0905.2506 [hep-ph].

[12] V. Mader \& R. Alkofer, PoS ConfinementX (2012), 063.

[13] D. Binosi, D. Ibáñez \& J. Papavassiliou, JHEP 1409 (2014), 019.

[14] A.K. Cyrol, M.Q. Huber \& L. von Smekal, Eur. Phys. J. C75 (2015), 102.

[15] A.K. Cyrol, L. Fister, M. Mitter, J.M. Pawlowski \& N. Strodthoff, Phys. Rev. D94 (2016), 054005 .

[16] R. Alkofer, C.S. Fischer, F.J. Llanes-Estrada \& K. Schwenzer, Annals Phys. 324 (2009), 106.

[17] R. Alkofer \& M. Mitter, PoS ConfinementX (2012), 061.

[18] G. Curci \& R. Ferrari, Nuovo Cim. A32 (1976), 151.

[19] T. van Ritbergen, A.N. Schellekens \& J.A.M. Vermaseren, Int. J. Mod. Phys. A14 (1999), 41.

[20] S. Laporta, Int. J. Mod. Phys. A15 (2000), 5087.

[21] A.D. Kennedy, J. Math. Phys. 22 (1981), 1330. 
[22] A. Bondi, G. Curci, G. Paffuti \& P. Rossi, Ann. Phys. 199 (1990), 268.

[23] A.N. Vasil'ev, M.I. Vyazovskii, S.É. Derkachov \& N.A. Kivel, Theor. Math. Phys. 107 (1996), 359.

[24] A.N. Vasil'ev, S.É. Derkachov \& N.A. Kivel, Theor. Math. Phys. 103 (1995), 487.

[25] A.N. Vasil'ev, M.I. Vyazovskii, S.É. Derkachov \& N.A. Kivel, Theor. Math. Phys. 107 (1996), 710.

[26] J.A. Gracey, JHEP 1209 (2012), 052.

[27] M.I. Buchoff \& M. Wagman, Phys. Rev. D93 (2016), 016005.

[28] P. Nogueira, J. Comput. Phys. 105 (1993), 279.

[29] J.A.M. Vermaseren, math-ph/0010025.

[30] M. Tentyukov \& J.A.M. Vermaseren, Comput. Phys. Commun. 181 (2010), 1419.

[31] C. Studerus, Comput. Phys. Commun. 181 (2010), 1293.

[32] A.I. Davydychev, J. Phys. A25 (1992), 5587.

[33] N.I. Ussyukina \& A.I. Davydychev, Phys. Atom. Nucl. 56 (1993), 1553.

[34] N.I. Ussyukina \& A.I. Davydychev, Phys. Lett. B332 (1994), 159.

[35] N.I. Ussyukina \& A.I. Davydychev, Phys. Lett. B305 (1993), 136.

[36] G. 't Hooft, Nucl. Phys. B190 (1981), 455.

[37] A.S. Kronfeld, G. Schierholz \& U.J. Wiese, Nucl. Phys. B293 (1987), 461.

[38] A.S. Kronfeld, M.L. Laursen, G. Schierholz \& U.J. Wiese, Phys. Lett. B198 (1987), 516. 